\title{
The SLC7A11: sperm mitochondrial function and non-canonical glutamate metabolism
}

\author{
José Manuel Ortiz-Rodríguez , Francisco Eduardo Martín-Cano, Gemma Gaitskell-Phillips , \\ Antonio Silva ${ }^{3}$, José Antonio Tapia ${ }^{2}$, Maria Cruz Gil ${ }^{1}$, Eloy Redondo ${ }^{1}$, Javier Masot ${ }^{1}$, \\ Cristina Ortega-Ferrusola ${ }^{1}$ and Fernando J Peña ${ }^{1}{ }^{1}$ \\ ${ }^{1}$ Laboratory of Equine Reproduction and Equine Spermatology, Veterinary Teaching Hospital, University of \\ Extremadura, Cáceres, Spain, ${ }^{2}$ Department of Physiology, University of Extremadura, Cáceres, Spain and ${ }^{3}$ Facility for \\ Innovation and Analysis in Animal Source Foodstuffs, University of Extremadura, Cáceres, Spain
}

Correspondence should be addressed to F J Peña; Email: fjuanpvega@unex.es

\begin{abstract}
Spermatozoa are redox-regulated cells, and stallion spermatozoa, in particular, present an intense mitochondrial activity in which large amounts of reactive oxygen species (ROS) are produced. To maintain the redox potential under physiological conditions, sophisticated mechanisms ought to be present, particularly in the mitochondria. In the present study, we investigated the role of the SLC7A11 antiporter. This antiporter exchanges intracellular glutamate for extracellular cystine. In the spermatozoa, cystine is reduced to cysteine and used for GSH synthesis. The importance of the antiporter for mitochondrial functionality was studied using flow cytometry and UHPLC/MS/MS approaches. Intracellular GSH increased in the presence of cystine, but was reduced in the presence of Buthionine sulphoximine (BSO), a $\gamma$-glutamylcysteine synthetase inhibitor $(P<\mathbf{0 . 0 0 1})$. Inhibition of the SLC7A11 antiporter with sulfasalazine caused a dramatic drop in intracellular GSH $(P<0.001)$ and in the percentage of spermatozoa showing active mitochondria $(P<\mathbf{0 . 0 0 1})$. These findings suggest that proper functionality of this antiporter is required for the mitochondrial function of spermatozoa. We also describe that under some conditions, glutamate may be metabolized following non-conventional pathways, also contributing to sperm functionality. We provide evidences, that the stallion spermatozoa have important metabolic plasticity, and also of the relation between redox regulation and metabolic regulation. These findings may have important implications for the understanding of sperm biology and the development of new strategies for sperm conservation and treatment of male factor infertility.

Reproduction (2020) 160 803-818
\end{abstract}

\section{Introduction}

Insemination with refrigerated semen is common practice in the equine industry (Vidament et al. 2012). In spite of the importance of this technique, unresolved questions still persist. In particular, the metabolism of the spermatozoa under this and other conditions is far from being fully understood. Nevertheless, significant advances have occurred in the past decade that have deepened our knowledge of stallion sperm physiology. Regarding this particular aspect, knowledge of the role of reactive oxygen species (ROS) has dramatically evolved (Salicioni et al. 2007, Gibb et al. 2014, O'Flaherty 2015, Lee et al. 2017, O'Flaherty \& Matsushita-Fournier 2017, Griffin et al. 2019, Ortega-Ferrusola et al. 2019, Pena et al. 2019). For a long time, the predominant belief was that ROS were merely toxic byproducts of metabolism that should be kept to a minimum with the use of antioxidants. However, paradoxical findings showing that more fertile spermatozoa were characterized by more intense ROS production, have challenged this paradigm (Gibb et al. 2014, Darr et al. 2016). Recent proteomic studies have widened our knowledge of sperm metabolism and have put assumed paradigms in spermatology into doubt (Amaral et al. 2013, 2014, Paiva et al. 2015, Swegen et al. 2015). In line with these recent findings, the relationship between metabolism and redox regulation is unveiled as a promising approach to improve current techniques for sperm conservation (Gibb et al. 2015, Darr et al. 2016, Swegen et al. 2016).

Reactive oxygen species are formed by incomplete reduction of molecular oxygen and are atoms or molecules with a single unpaired electron. These include, among others, superoxide $\left(\mathrm{O}_{2}{ }^{-}\right)^{-}$, the hydroxyl radical $\left(\mathrm{OH}^{-}\right)$and the lipid peroxide radical (LOO). Although hydrogen peroxide $\left(\mathrm{H}_{2} \mathrm{O}_{2}\right)$ itself is not a free radical, it is a precursor of free radicals. $U V$ radiation and the presence of metal ions $\left(\mathrm{Fe}^{2+}\right.$, $\mathrm{Fe}^{3+}$ or $\mathrm{Cu}^{2+}$ ) generate hydroxyl radicals (Pena 
et al. 2019). All aerobic organisms depend on the generation of ATP from the four-electron reduction of molecular oxygen into water. During this process, the mitochondrial transport chain may lose electrons leading to the formation of ROS. Mitochondrial dysfunction may exacerbate the loss of electrons and thus increase the production of reactive oxygen species (Kalyanaraman 2013). Stallion spermatozoa are characterized by an intense mitochondrial activity in comparison with other mammals (Gibb et al. 2014, Darr et al. 2016, Griffin et al. 2019). Recent research points to sophisticated regulation of production and elimination of ROS, with important roles in stallion sperm physiology. The reversible oxidation of thiolcontaining residues in specific amino acids of regulatory proteins is of particular importance (OrtegaFerrusola et al. 2019, Ortiz-Rodriguez et al. 2019b). Mitochondria plays a major role in the production of reactive oxygen and nitrogen species in spermatozoa (Ortega Ferrusola et al. 2009, Davila et al. 2016), and thus also play a role in redox regulation. Recently, it was found that the SLC7A11 antiporter has a role in redox regulation in the spermatozoa; incorporating cystine (the oxidized form of cysteine) in exchange for intracellular glutamate (Ortiz-Rodriguez et al. 2019b). This is a mechanism which maintains intracellular GSH at physiological levels (Ortega-Ferrusola et al. 2019). Recycling oxidized GSH (GSSG) requires reducing equivalents that are produced in energy metabolism. Glutathione is synthesized exclusively in the cytosol, but is distributed to the mitochondria through specific carriers with the end result that GSH concentration in the mitochondrial matrix is equal to that of the cytosol (Ribas et al. 2014). Here GSH acts as an electron donor as part of a defense mechanism against respiration induced ROS and also participates in the detoxification of lipid hydroperoxides. Moreover, mitochondrial GSH is a regulator of mitochondrial permeability, and thus of cell death (Coppola \& Ghibelli 2000, Circu et al. 2008). However, inhibition of the antiporter may lead both to positive and negative effects in stallion spermatozoa (OrtizRodriguez et al. 2019b). This apparently paradoxical effect may relate to other functions of the antiporter, not only as a source of extracellular cystine used for GSH synthesis. Recently SLC7A11 has been described as having a possible role in glutamate metabolism. In some cellular models, disruption of the SLC7A11 antiporter greatly improves cell viability after glucose withdrawal, because conservation of glutamate enables cells to maintain mitochondrial respiration (Shin et al. 2017). To test if a similar picture may be occurring in stallion spermatozoa, we investigated the SLC7A11 antiporter, paying special attention to the effects on mitochondrial function due to the central role of these organelles in redox regulation and metabolism.

\section{Materials and methods}

\section{Reagents and media}

L-Cystine (Cyss), L-buthionine-sulfoximine (BSO), monochlorobimane (MCB), sulfasalazine (SS), $\alpha$-methyl4-carboxyphenilglycine (mCPG), R162 (GDH1 inhibitor), methyl $\alpha$-ketoglutarate, Erastin, RSL3, liproxstatin-1 and all other chemicals were purchased from Sigma Aldrich. All other reagents for flow cytometry were purchased from Thermofisher. Anti-GDH1 antibody was purchased from Abcam (ab89967).

\section{Semen collection and processing}

Semen was collected from 11 stallions maintained according to institutional and European animal care regulations (Law 6/2913 June 11th and European Directive 2010/63/EU). All procedures used in this study received approbation from the ethical committee of the University of Extremadura (Ref\# AGL201783149R). Ejaculates were collected using a prewarmed, lubricated Missouri model artificial vagina following standard veterinary practices. After collection, the ejaculate was immediately evaluated and processed in the adjacent laboratory. Colloidal centrifugation (Morrell et al. 2011) was performed to remove dead spermatozoa and contaminating particles from the ejaculate. The pellet was re-extended to a final concentration of $40 \times 10^{6}$ spermatozoa $/ \mathrm{ml}$ in Tyrodes media $\left(96 \mathrm{mM} \mathrm{NaCl}, 3.1 \mathrm{mM} \mathrm{KCl}, 2 \mathrm{mM} \mathrm{CaCl} \cdot 2 \mathrm{H}_{2} \mathrm{O}, 0.4\right.$ $\mathrm{mM} \mathrm{MgSO} \cdot 7 \mathrm{H}_{2} \mathrm{O}, 0.3 \mathrm{mM} \mathrm{KH_{2 }} \mathrm{PO}_{4}, 20 \mathrm{mM}$ HEPES, $5 \mathrm{mM}$ glucose, $21.7 \mathrm{mM}$ Na lactate, $1 \mathrm{mM} \mathrm{Na}$ Pyruvate, $15 \mathrm{mM}$ $\mathrm{NaHCO}_{3}, 3 \% \mathrm{v} / \mathrm{v}$ BSA, $50 \mathrm{mg} / \mathrm{mL}$ kanamicin) pH 7.4 (Balao da Silva et al. 2014). The sperm suspension was then split into sub-samples for control and experimental treatments. For UHPLC/MS/MS experiments ejaculates were also processed through colloidal centrifugation to remove dead and contaminating cells and separate seminal plasma. Pellets obtained were washed three times in PBS (1500 $\mathrm{g} \times 10^{\prime}$ at $4^{\circ} \mathrm{C}$ ) suspended in $1 \mathrm{~mL}$ of PBS and stored at $-80^{\circ} \mathrm{C}$ until further use.

\section{Flow cytometry}

Flow cytometry analyses were conducted using a Cytoflex ${ }^{\circledR}$ flow cytometer (Beckman Coulter) equipped with violet, blue, yellow and red lasers. The instrument was calibrated daily using specific calibration beads provided by the manufacturer. A compensation overlap was performed before each experiment. Files were exported as FCS files and analyzed using FlowjoV 10.6.1 Software (Ashland, OR, USA). Unstained, singlestained, and Fluorescence Minus One (FMO) controls were used to determine compensations and positive and negative events, as well as to set regions of interest as described in previous publications by our laboratory (Gallardo Bolanos et al. 2014, Martin Munoz et al. 2015).

\section{Measurement of GSH, viability and mitochondrial membrane potential in stallion spermatozoa}

Intracellular GSH was measured by adapting previously published protocols optimized for GSH detection using flow 
cytometry (Capek et al. 2017) adapted to equine spermatozoa in our laboratory (Ortiz-Rodriguez et al. 2019b). The mitochondrial membrane potential and sperm viability were also simultaneously assessed. In brief, sperm aliquots (1-5 x $10^{6} \mathrm{sperm} / \mathrm{mL}$ ) were stained with JC-1 $1 \mu \mathrm{M}$ (30 min in the darkness at r.t.), DRAQ7 $3 \mu \mathrm{M}$ and monochlorobimane (MCB) $10 \mu \mathrm{M}$ (10 $\mathrm{min}$ in the darkness at r.t). The applied gating strategy is depicted in Fig. 1. Briefly, after assessment of the quality of the flow, doublets and debris were gated out, MCB was detected at a peak excitation of $405 \mathrm{~nm}$ and emission of 450/45 nm BP, JC- 1 was detected at a peak excitation of 511 $\mathrm{nm}$ and emission of $596 \mathrm{~nm}$ (aggregates) and DRAQ7, at a peak excitation of 640 , and emission of $690 \mathrm{~nm}$.

\section{Computational flow cytometry (t-SNE)}

The data from each multi-parametric assay described in the 'Material and methods' section were exported as FCS files from the flow cytometer and loaded and analyzed using FlowjoV 10.6.2 Software (Ashland, OR, USA). For computational analysis, data from all the replicates for each treatment were concatenated and single cell events analyzed. The files obtained after concatenation were exported as FCS files. Flow cytometry files were then analyzed using non-linear dimensional reduction techniques (t-SNE) and automatic classification of cellular expression by non-linear
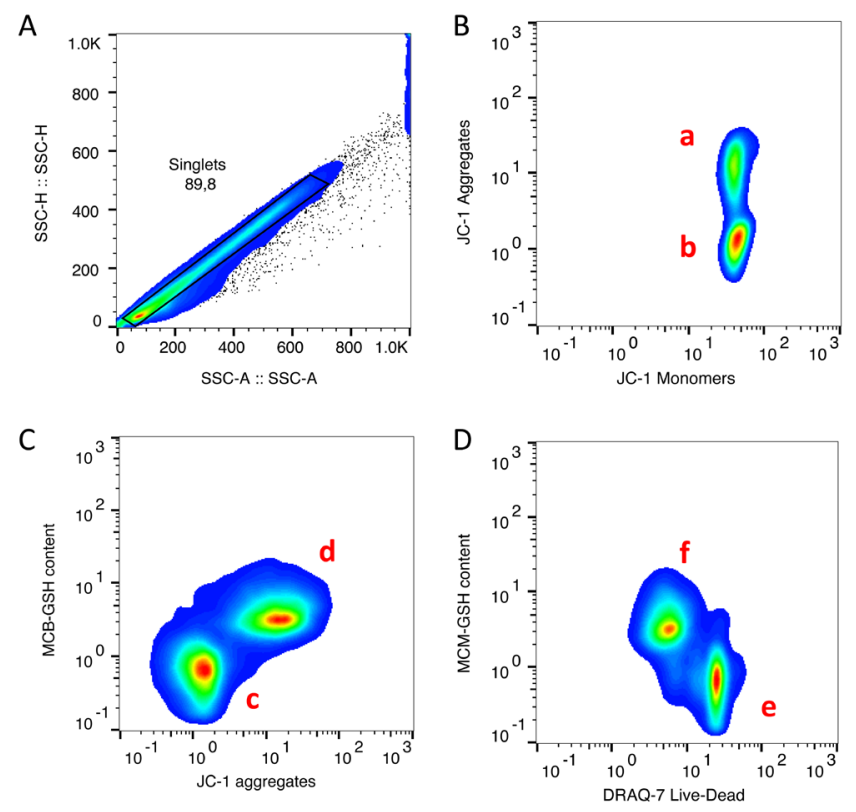

Figure 1 Gating strategy for flow cytometry-based determination of changes in GSH, mitochondrial activity and percentages of live cells in stallion spermatozoa. After verification of the quality of the flow, single cells were identified confronting SSC-H vs SSC-A gating out doublets (A). Then the percentages of cells showing high (a) and low (b) mitochondrial activity (B), the relative GSH content (C) and the percentage of live spermatozoa (D) were identified and quantified. Interestingly most the population of spermatozoa showing high mitochondrial activity (a) was also characterized by high GHS content (d) showing the close link between mitochondrial activity and GSH. stochastic embedding (ACCENSE) accessible at http://www. cellaccense.com/index.html. The Barnes-Hut implementation of t-SNE was used for low dimensional embedding to perform dimensional reduction of cytometry data, classification of cells was based on $\mathrm{K}$ means techniques with the significance level set at $P=0.0001$. ACCENSE identifies clusters within multidimensional data without losing single cell resolution (Shekhar et al. 2014), allowing automatic gating of cells.

\section{UHPLC-MS/MS detection of reduced and oxidized glutathione}

The reduced (GSH) and oxidized (GSSG) forms of glutathione were analyzed by UHPLC-MS/MS following previously published protocols (Ortega-Ferrusola et al. 2019). In brief, a chromatography separation was performed using a Thermo Ultimate 3000 Ultra (Thermo Fisher Scientific) and separations were carried out on a C18 column $(100 \times 21 \mathrm{~mm} ; 1.7 \mu \mathrm{m}$, Bruker Intensity Solo, Billerica, MA, USA). The analytes eluted from the UHPLC column were directly introduced in an ion trap mass spectrometer (Amazon SL, Bruker Daltonik GmbH, Bremen, Germany). The mass spectrometer was run in MS/MS mode, in which the protonated reduced glutathione $(308 \mathrm{~m} / \mathrm{z})$ and protonated oxidized glutathione $(613 \mathrm{~m} / \mathrm{z})$ were isolated and subjected to collision-induced dissociation, using Helium gas and radio frequency (RF) with fragmentation amplitudes of 0.45 and $0.65 \mathrm{~V}$, respectively. The full scan MS/MS or MS2 spectra was obtained under these conditions, in which the $178.6 \mathrm{~m} / \mathrm{z}$ ion was selected as the quantification ion for reduced glutathione and $484+355 \mathrm{~m} / \mathrm{z}$ was selected as the quantification ion for oxidized glutathione.

\section{Metabolomics}

The sperm pellets were redissolved in $1 \mathrm{~mL}$ milliQ water and sonicated for $3 \mathrm{~s}$. Immediately afterwards, samples were centrifuged at $4,000 \mathrm{~g}$ at $4{ }^{\circ} \mathrm{C}$ for $3 \mathrm{~min}$ and the supernatant was injected into the UHPLC-MS/MS. Separation and analysis of the samples were performed with a UHPLC/MS system consisting of an Agilent 1290 Infinity II Series HPLC (Agilent Technologies) equipped with an automated multisampler module and a High Speed Binary Pump, coupled to an Agilent 6550 Q-TOF Mass Spectrometer (Agilent Technologies) using an Agilent Jet Stream Dual electrospray (AJS-Dual ESI) interface. Control of the HPLC and Q-TOF was through the MassHunter Workstation Data Acquisition software (Agilent Technologies, Rev. B.08.00). Samples were injected onto an Agilent C18 HPLC column $(4.6 \mathrm{~mm}, 100 \mathrm{~mm} \times 2.1 \mu \mathrm{m}$, Agilent technologies), thermostatted at $40^{\circ} \mathrm{C}$, at a flow rate of $0.4 \mathrm{ml} / \mathrm{min}$. The injection volume was $5 \mu \mathrm{L}$. For gradient elution, solvent $\mathrm{A}$ was: $0.2 \%(\mathrm{v} / \mathrm{v})$ acetic acid in Milli $\mathrm{Q}$ water and solvent $B$ was $0.2 \%$ acetic acid in acetonitrile. In the beginning, solvent B was maintained at $2 \%$ for 3 min. Solvent B was increased from 0 to $98 \%$ from 4 to $12 \mathrm{~min}$ and held at $98 \%$ for an additional $2 \mathrm{~min}$. Then solvent B was returned to the initial conditions up to min 15. The mass spectrometer was operated in positive and negative modes. The best results were obtained in negative mode. The nebulizer gas pressure was set to 35 psi, whereas the drying gas flow was set to 
$12 \mathrm{~L} / \mathrm{min}$ at a temperature of $150^{\circ} \mathrm{C}$, and the sheath gas flow was set to $15 \mathrm{~L} / \mathrm{min}$ at a temperature of $350^{\circ} \mathrm{C}$. The capillary spray, fragmentor and octopole RF Vpp voltages were 3500, 360 and $750 \mathrm{~V}$, respectively. Centroid data were acquired for both MS and MS/MS scans in extended dynamic range mode. MS and MS/MS mass range was 50-950 m/z and scan rates were 5 spectra/s for MS and 2 spectra/s for MS/ MS. All lons mode was used without precursor selection in two experiments or channels at the same time, one of low energy ( $0 \mathrm{~V}$ collision energy) and one of high energy (20 V collision energy). The low value produces the precursor ions (MS scan) for the compounds and the high value generates the precursors plus their product ions (MS/MS scan). Data processing and analysis were performed using MassHunter Qualitative Analysis Software (Rev B.07.00.201, Agilent Technologies). The chromatography peaks were qualified by accurate mass precursor ions and accurate mass product ions from the MS/MS spectrum. Furthermore, some compounds were also identified using the METLIN metabolites Personal Compounds Database Library (PDCL) (Agilent Technologies), in which mass peak in the low energy channel are first searched for against this PDCL for compounds which have the same $\mathrm{m} / \mathrm{z}$ values. In addition, the fragment ions in the high energy channel for these compounds were compared with MS/MS spectra from PDCL.

\section{Measurement of oxidation-reduction potential}

Oxidation-reduction potential (ORP) was measured using the RedoxSYS ${ }^{\circledR}$ diagnostic system (Englewood CO, USA), following previously published protocols adapted for equine spermatozoa (Ortiz-Rodriguez et al. 2019a).

\section{Western blotting (WB)}

To separate the proteins according to their apparent molecular masses, SDS-PAGE was performed as previously described (Aparicio et al. 2016). In brief, proteins were extracted and denatured by boiling for $10 \mathrm{~min}$ at $70^{\circ} \mathrm{C}$ in a loading buffer supplemented with 5\% mercaptoethanol. Ten micrograms of sperm protein extract were loaded and resolved by SDSPAGE on a $10 \%$ polyacrylamide gel. Immunoblotting was performed by incubating the membranes in blocking buffer at $4^{\circ} \mathrm{C}$ overnight with of $1 \mu \mathrm{g} / \mathrm{mL}$ anti GDH-1 primary antibody.

\section{Immunocytochemistry (ICC)}

Indirect immunofluorescence was performed as previously described (Tapia et al. 2012). After blocking, cells were incubated with primary antibody anti GDH-1, overnight at $4{ }^{\circ} \mathrm{C}$ diluted $1 / 200$ in PBS containing 5\% BSA (w/v). The following day, cells were washed with PBS and further incubated for 45 min at r.t. with goat anti-goat IgG antibody conjugated with Alexa Fluor 647 diluted to $1 / 500$ in PBS containing 5\% BSA $(w / v)$. Finally, cells were thoroughly washed with PBS. A total of 5000 cells were analyzed in the ImageStream X Mark II Imaging Flow Cytometer (Merck Millipore) using a 642 nm line laser with intensity set to $100 \mathrm{~mW}$, at $60 \times$ magnification. Data analysis of the raw images was accomplished using
IDEAS1software (Version 6.0.309). The absence of nonspecific staining was measured by processing the samples without primary antibody (secondary antibody only).

\section{Computer-assisted sperm analysis (CASA)}

Sperm motility and velocity were assessed using a computerassisted sperm analysis (CASA) system (ISAS Proiser, Valencia, Spain) according to standard protocols used at our center (Ortega-Ferrusola et al. 2009). Semen samples were loaded in a Leja ${ }^{\circledR}$ chamber with a depth of $20 \mu \mathrm{m}$ (Leja, Amsterdam, The Netherlands) and placed on a stage warmed at $37^{\circ} \mathrm{C}$. Analysis was based on an evaluation of 60 consecutive digitized images obtained using a $10 \times$ negative phase-contrast objective (Olympus CX 41). At least 500 spermatozoa per sample were analyzed in random fields. Spermatozoa VAP $>35 \mu \mathrm{m} / \mathrm{s}$ were considered motile. Spermatozoa deviating $<45 \%$ from a straight line were classified as linearly motile.

\section{Assessment of caspase 3 activity and phosphatidylserine (PS) translocation}

Annexin V 647 conjugated and CellEvent ${ }^{\circledR}$ Caspase 3/7 Green Detection Reagent were combined in a multi-parametric test and evaluated by FC (Ortega-Ferrusola et al. 2017). Samples were loaded with Hoechst $33342(0.3 \mu \mathrm{M})$ and CellEvent $(2 \mu \mathrm{M})$ and incubated at room temperature for $15 \mathrm{~min}$. Following this, the samples were washed by a short centrifugation spin for $12^{\prime \prime}$ and suspended in $200 \mu \mathrm{L}$ of Annexin binding-buffer (solution in $10 \mathrm{mM}$ HEPES, $140 \mathrm{mM} \mathrm{NaCl}, 2.5 \mathrm{mM} \mathrm{CaCl}_{2}, \mathrm{pH}$ 7.4). Five microliters of Annexin $V$ was added to $200 \mu \mathrm{L}$ of sample. After $15 \mathrm{~min}$ of incubation, in the dark at room temperature, 400 $\mu \mathrm{L}$ of $1 \times$ Annexin binding-buffer was added before reading in the flow cytometer (Cytoflex ${ }^{\circledR}$ flow cytometer, Beckman Coulter). To gate dead spermatozoa samples were stained with $0.3 \mu \mathrm{M}$ of Eth-1 and incubated for $5 \mathrm{~min}$ before they were immediately evaluated in a flow cytometer (Cytoflex ${ }^{\circledR}$ flow cytometer, Beckman Coulter). CellEvent staining was validated as previously described (Ortiz-Rodriguez et al. 2019a).

\section{Statistical analysis}

Sperm samples were obtained from 11 different stallions. All experiments were repeated at least three times with independent samples (three separate ejaculates from each of the donor stallions). The normality of the data was assessed using the Kolmogorov-Smirnoff test. Paired $t$-tests and oneway ANOVA followed by Dunnett's multiple comparisons test and were performed using GraphPad Prism version 7.00 for www.graphpad.com. Differences were considered significant when $P<0.05$. Results are displayed as means \pm S.E.M.

\section{Results}

\section{Stallion spermatozoa synthetize GSH from cystine}

In order to confirm changes in intracellular glutathione in both reduced (GSH) and oxidized (GSSG) forms, concentrations were monitored using UHPLC/MS/MS. 
Incubation of stallion spermatozoa in the presence of $0.5 \mathrm{mM}$ Cyss caused an increase in intracellular GSH that was prevented with coincubation in the presence of buthionine sulphoximine (BSO $50 \mu \mathrm{M}$ ) a $\gamma$-glutamylcysteine synthetase inhibitor that is the limiting enzyme for GSH synthesis (Fig. 2A). At the same time, levels of oxidized glutathione (GSSG) were observed to be reduced after $1 \mathrm{~h}$ of incubation in Cyss treated samples (Fig. 2B).

\section{Inhibition of the SLC7A11 antiporter reduces stallion sperm viability}

The incubation of stallion spermatozoa in the presence of the SLC7A11 antiporter inhibitor sulfasalazine (SS) reduced sperm viability at $200 \mu \mathrm{M}(P<0.01)$ and 500 $\mu \mathrm{M}(P<0.001)$. The percentages of live spermatozoa were reduced from $71.0 \pm 4.4 \%$ in controls to $64.1 \pm 4.4$ and $55.5 \pm 5.4 \%$, respectively, in the presence of SS at 200 and $500 \mu \mathrm{M}$. The simultaneous presence of Cyss and SS 200 and $500 \mu \mathrm{M}$ also caused reduced sperm
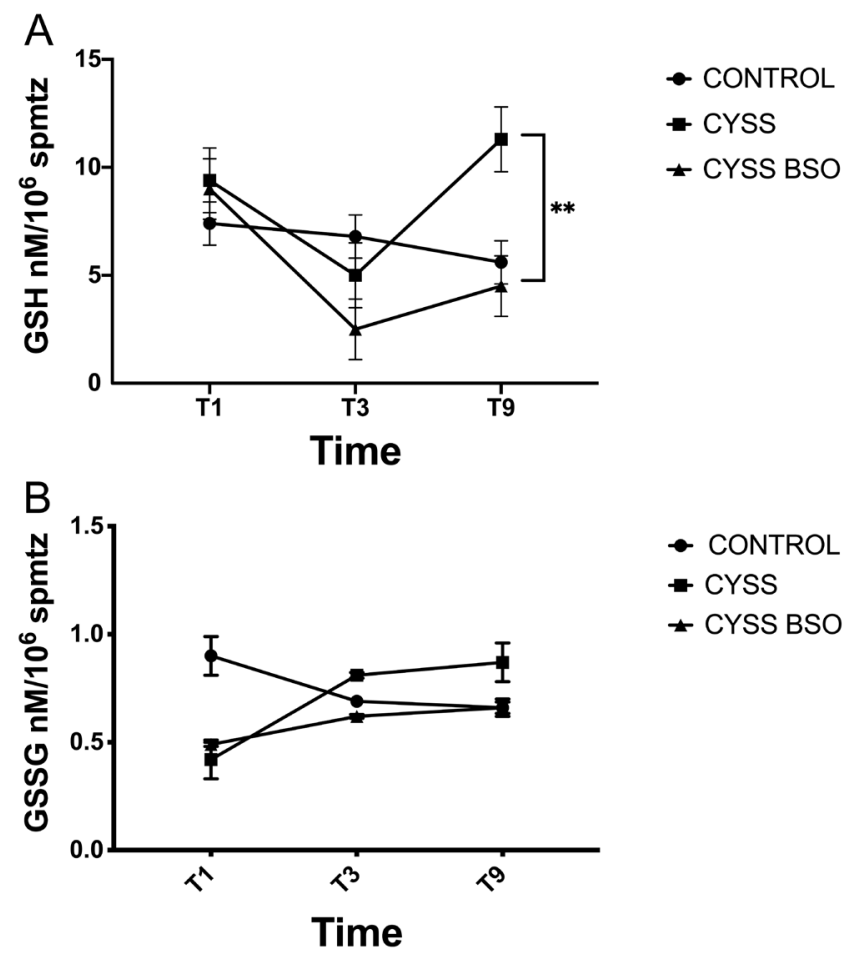

Figure 2 Stallion spermatozoa incorporate Cyss for GSH synthesis. In order to determine the functionality of the SLC7A11 antiporter, stallion spermatozoa were incubated in the presence of Cys or Cys and BSO (inhibitor of $\gamma$-glutamylcysteine synthetase a key enzyme in glutathione biosynthesis). Then the content of reduced (GSH) and oxidized (GSSG) glutathione was investigated using mass spectrometry (MS) as described in the 'Materials and methods' section. Incubation in the presence of Cyss increased intracellular $\mathrm{GSH}(P<0.01)$, effect that was prevented in the presence of BSO (A). The changes in GSSG are presented in B. Data are presented as means \pm S.E.M. of five different replicate experiments, ${ }^{* *} P<0.01$. viability $(P<0.01$ and $P<0.001$, respectively) (Fig. $3 \mathrm{~A})(68.5 \pm 3.7$ in controls to $53.0 \pm 5.4$ in Cyss-SS $500 \mu \mathrm{M}$ supplemented sub-samples). When stallion spermatozoa were incubated in the presence of the inhibitor $\alpha$-methyl-4-carboxyphenilglycine (mCPG), all concentrations tested reduced viability $(P<0.05)$ (Fig. 3B), from $63.0 \pm 5.9 \%$ in controls to $59.7 \pm 7.0$, $57.7 \pm 6.5$ and $54.8 \pm 7.0$ in samples treated with 100 , 200 and $500 \mu \mathrm{M} \mathrm{mCPG}$, respectively, an effect that was prevented by Cyss $(0.5 \mathrm{mM})$ when $\mathrm{mCPG}$ was present at 100 and $200 \mu \mathrm{M}$ (Fig. 3B).

\section{Inhibition of the SLC7A11 antiporter with sulfasalazine reduces mitochondrial membrane potential $(\Delta \Psi m)$}

Semen samples incubated in the presence of SS experimented a drop in the percentage of spermatozoa showing high $\Delta \Psi \mathrm{m}$ from $46.3 \pm 5 \%$ of the spermatozoa in controls to $23.1 \pm 4.5 \%$ in the presence of SS $500 \mu \mathrm{M}$ $(P<0.01)$. Cyss showed a synergistic effect, reducing the percentage of spermatozoa with high $\Delta \Psi \mathrm{m}$ in samples incubated in the presence of $0.5 \mathrm{mM}$ Cyss and $500 \mu \mathrm{M}$ SS $(P<0.001)$ to $12.6 \pm 2 \%$. Also, SS $200 \mu \mathrm{M}$ in the presence of Cyss resulted in a reduced percentage of spermatozoa with high $\Delta \Psi \mathrm{m}(35.5 \pm 4 P<0.01)$ (Fig. 4-1A). When the second inhibitor was used no effect was observed, to the contrary, a combination of 0.5 $\mathrm{mM}$ Cyss and $100 \mu \mathrm{M}$ mCPG resulted in an increased percentage of spermatozoa with high $\Delta \Psi \mathrm{m}(P<0.001)$, from $40.0 \pm 1.4$ in controls to $50.1 \pm 1.8$ in samples supplemented with $0.5 \mathrm{mM}$ Cyss and mCPG $100 \mu \mathrm{M}$ (Fig. 4-2A).

\section{Sulfasalazine reduces intracellular GSH, while carboxyphenylglicine increases intracellular GSH and reduces the steady state redox potential (Eh) in presence of Cyss}

Since a close relationship has been established between GSH content and sperm viability, changes in intracellular GSH were monitored. Incubation of stallion spermatozoa in the presence of $500 \mu \mathrm{M}$ SS reduced intracellular GSH content to $72 \%$ of the initial values $(P<0.01)$ (Fig. 5A), an effect that was also seen in the presence of $0.5 \mathrm{mM}$ Cyss. To the contrary, $500 \mu \mathrm{M}$ mCPG induced a $28.81 \%$ increase in GSH. This effect was even more evident in the presence of $0.5 \mathrm{mM}$ Cyss and $100 \mu \mathrm{M}$ mCPG with an increase of $48,22 \%$ with respect to initial values (Fig. 5B). Concomitantly with increased intracellular GSH the combination of $0.5 \mathrm{mM}$ Cyss and $100 \mu \mathrm{M}$ mCPG reduced Eh $(P<0.05)$ (Fig. 5C). The t-SNE maps built after computational analysis of the data, showed how the treatments modified GSH content in the whole population of spermatozoa at the single cell level. As seen in Fig. 5E, 0.5 mM Cyss supplementation caused an increase in GSH content. At the right of the 

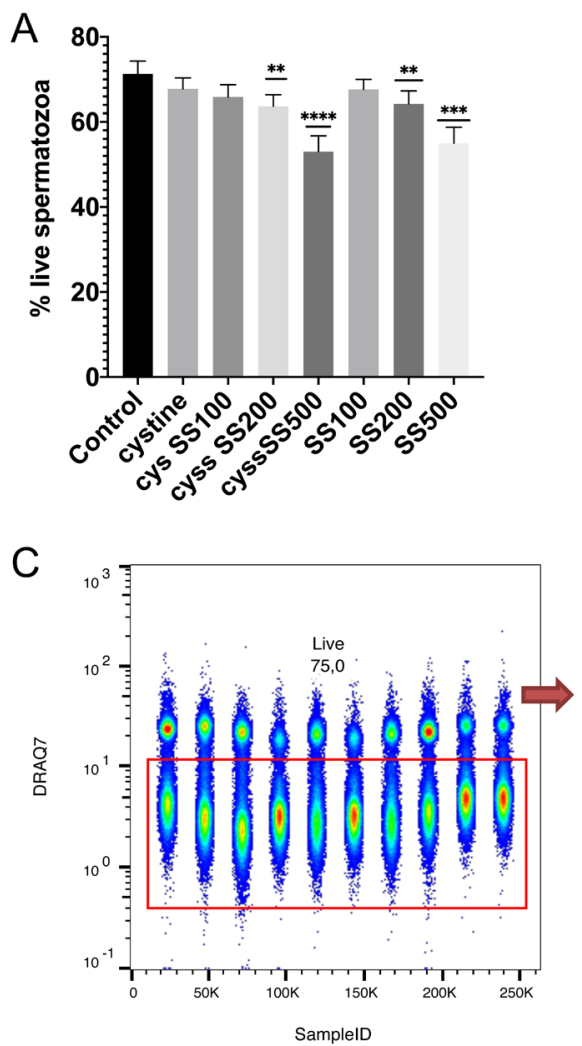
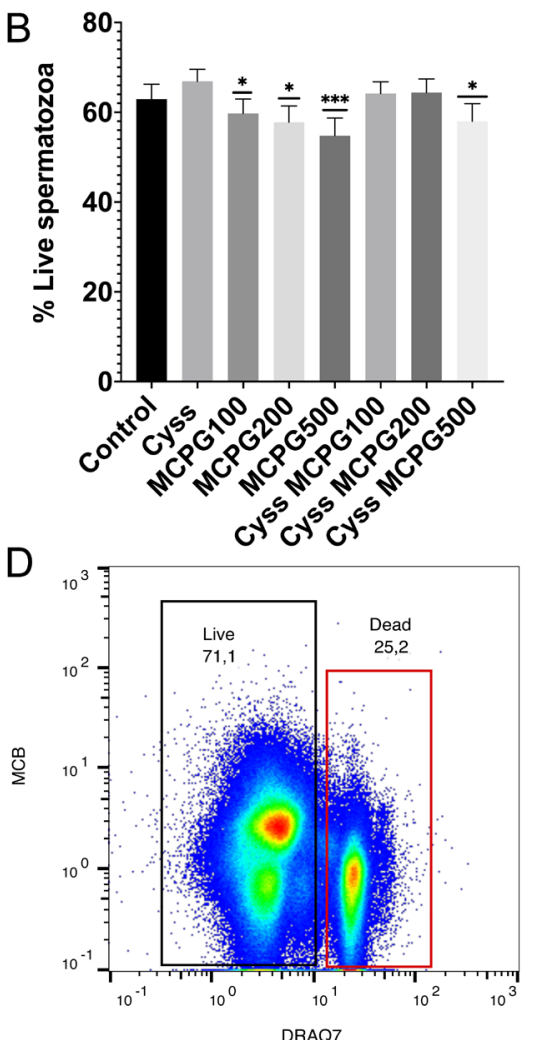

Figure 3 Inhibition of the SLC7A11 antiporter reduces sperm viability. To determine the impact of SLC7A11 on viability, stallion spermatozoa were incubated in the presence of SS and mCPG. SS reduced viability at 200 and $500 \mu \mathrm{M}(\mathrm{A})$. The incubation in the presence of $\mathrm{mCPG}$ reduced viability that was prevented by $0.5 \mathrm{mM}$ Cyss when mCPG was present at 100 and $200 \mu \mathrm{M}$ (B). Representative cytograms of the assay are presented in $\mathrm{C}$ and D. In C data from ten different replicates are concatenated (50,000 events per replicate), in $\mathrm{D}$ the cytogram represents all the events gathered in the ten replicates in a single 2D plot identifying live and dead cells. Data are means \pm S.E.M., ${ }^{* * *} P<0.001$. ${ }^{*} P<0.05$. $* * P<0.01$.
t-SNE map the color code for the heat map is depicted. Blue areas represent low expression, red, yellow and green increased expression. Supplementation with 0.5 $\mathrm{mM}$ Cyss and $100 \mu \mathrm{M}$ mCPG caused an overall increase in the content of GSH (Fig. 5F) seen as a reduction of the blue areas in the t-SNE map and an increase in yellowgreen and red areas. To the contrary, SS at $500 \mu \mathrm{M}$ caused a reduction of GSH content as seen in the $\mathrm{SNE}$ map (dominance of the blue color as seen in Fig. 5G).

\section{Erastin reduces sperm viability that is prevented by the ferroptosis inhibitor liproxstatin-1}

In order to determine if ferroptosis can be induced in ejaculated stallion spermatozoa, spermatozoa were treated with erastin $40 \mu \mathrm{M}$, a known inductor of ferroptosis, RSL3 $(10 \mu \mathrm{M}$ ) (an inhibitor of GPX-4) and SS $500 \mu \mathrm{M}$. Moreover, the specific ferroptosis inhibitor, liproxstatin-1 (5 and $10 \mu \mathrm{M}$ ) was also used. These concentrations were chosen on the basis of literature currently available in germinal cells (Bromfield et al. 2019). Incubation of stallion spermatozoa in the presence of erastin caused a significant reduction in the percentage of live spermatozoa after incubation for 3 $\mathrm{h}$ at $37^{\circ} \mathrm{C}$. From $37.4 \pm 2.8$ in controls to $25.6 \pm 2.0$ in erastin treated spermatozoa $(P<0.001)$ (Fig. 6A). This reduction was prevented by the presence of the ferroptosis inhibitor liproxstatin-1. We also determined the potential involvement of caspase 3 in sperm death.
The simultaneous presence of liproxstatin- 1 with erastin and SS reduced the percentage of caspase 3 positive cells (Fig. 6B) $(P<0.05)$. The percentage of dead cells were higher in the presence of erastin, liproxstatin-1 and in the simultaneous presence of erastin and liproxstatin-1 (Fig. 6C) $(P<0.05, P<0.01)$.

\section{Erastin reduces sperm motility, velocities and beat cross frequency (BCF), while $R S L$ reduces velocities and $B C F$}

A significant reduction was observed in the percentage of motile sperm, from $80.1 \pm 1.25$ in controls to $61.2 \pm 4.1$ in the presence of erastin $(P<0.001)$ (Fig. $7 A)$. The simultaneous presence of liproxstatin- 1 had a synergistic inhibitory effect, further reducing motility to $49.9 \pm 3.9 \%(P<0.001)$. RSL3 and SS had no effect, although RSL3 in the presence of liproxstatin-1 caused a reduction in motility $(P<0.001)$. A similar picture was observed when referring to percentages of linear motile spermatozoa (Fig. 7B). Interestingly, and in contrast to what was observed regarding motilities, both inducers of ferroptosis (erastin and RSL3) but not SS, reduced sperm velocities and BCF (Fig. 7D, E and F). Circular velocity was reduced from $213 \pm 5.4 \mu \mathrm{m} / \mathrm{s}$ in controls to $124.3 \pm 4.53 \mu \mathrm{m} / \mathrm{s}$ in samples treated with erastin $(P<0.001)$, and to $184.7 \pm 6.8 \mu \mathrm{m} / \mathrm{s}(P<0.05)$ in samples treated with RSL3 $(P<0.05)$. Liproxstatin-1 had no effects in the presence of erastin, but had a 
(1)

A

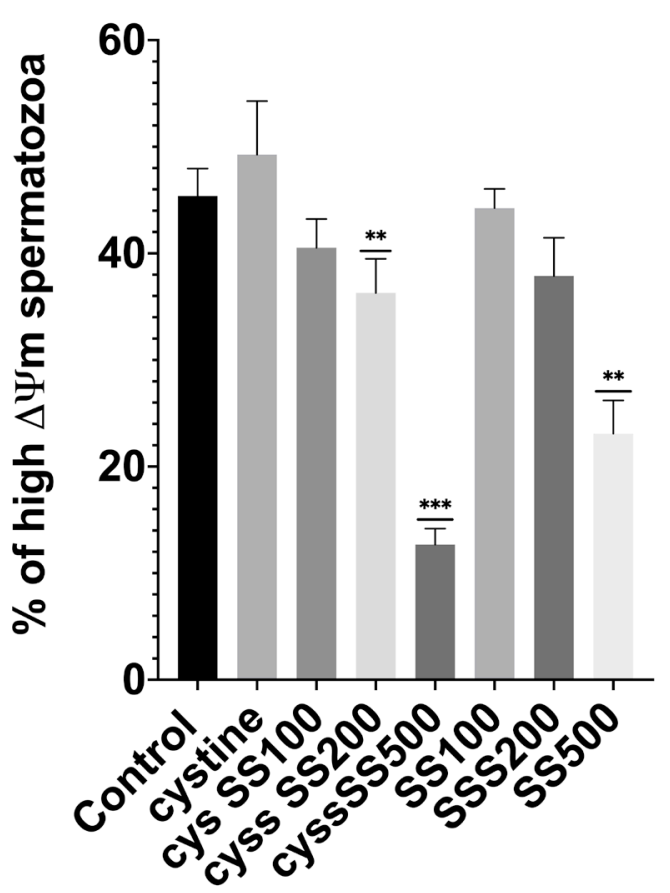

B
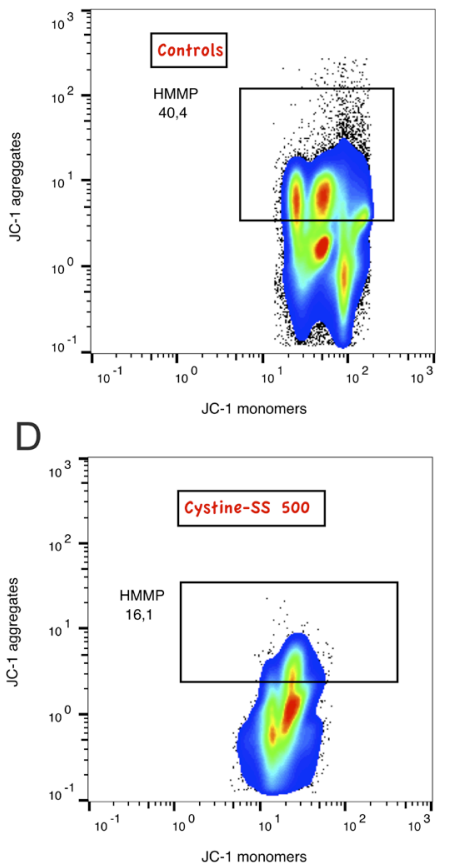

(2)

B

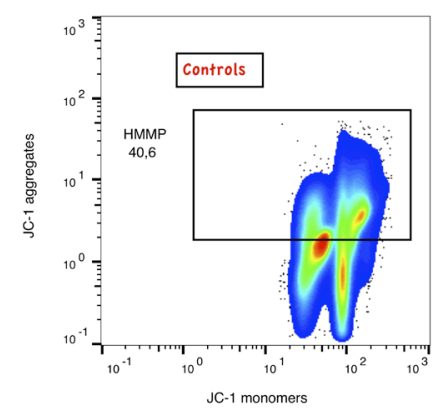

D

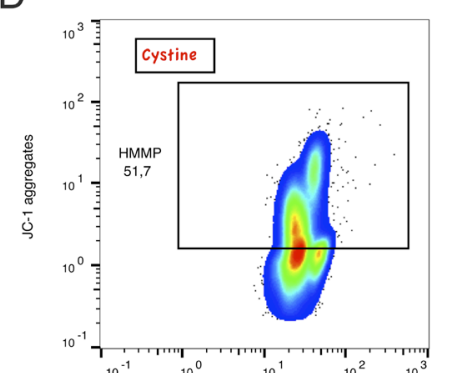

C
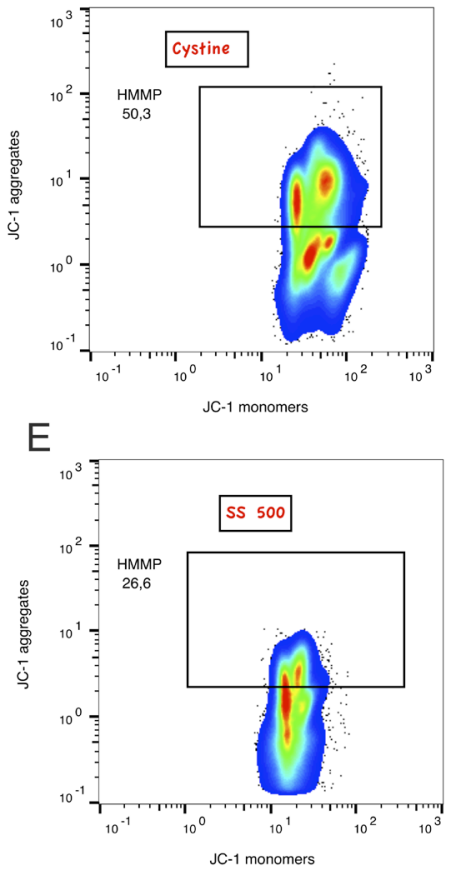

E
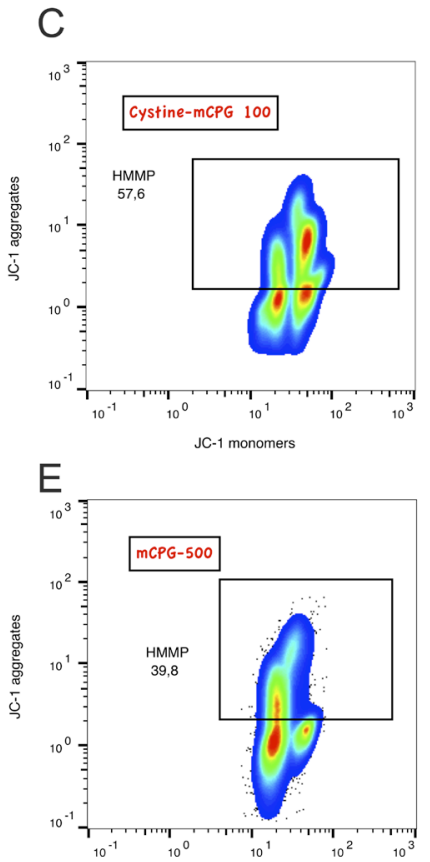

Figure 4 (1) (A) Sulfasalazine reduces mitochondrial membrane potential. Incubation of stallion spermatozoa in the presence of $500 \mu \mathrm{M}$ SS reduced mitochondrial membrane potential. Simultaneous presence of Cyss caused further decrease in mitochondrial membrane potential. (B, C and $\mathrm{D}$ ) representative $2 \mathrm{D}$ plots of concatenated replicates depicting the effect of the different treatments in the percentages of spermatozoa showing high mitochondrial membrane potential. (2) (A) mCPG does not reduce mitochondrial membrane potential. Stallion spermatozoa were incubated in presence of $\mathrm{mCPG}$ or $\mathrm{mCPG}$ and Cyss, and mitochondrial membrane potential evaluated after $3 \mathrm{~h}$ of incubation at $37^{\circ} \mathrm{C}$. $(\mathrm{B}, \mathrm{C}, \mathrm{D}$ and E) 2D plots after concatenation of all the replicates showing changes in the mitochondrial activity. In (C) (0.5 mM Cyss and mCPG $100 \mu \mathrm{M}$ supplemented samples) and in (D) (samples supplemented with $0.5 \mathrm{mM}$ Cyss) there is an increase in mitochondrial membrane potential. (B) are controls and (E) are samples supplemented with mCPG $500 \mu \mathrm{M}$. Data are means \pm S.E.M. ${ }^{* * *} P<0.001 .{ }^{* *} P<0.01$. 

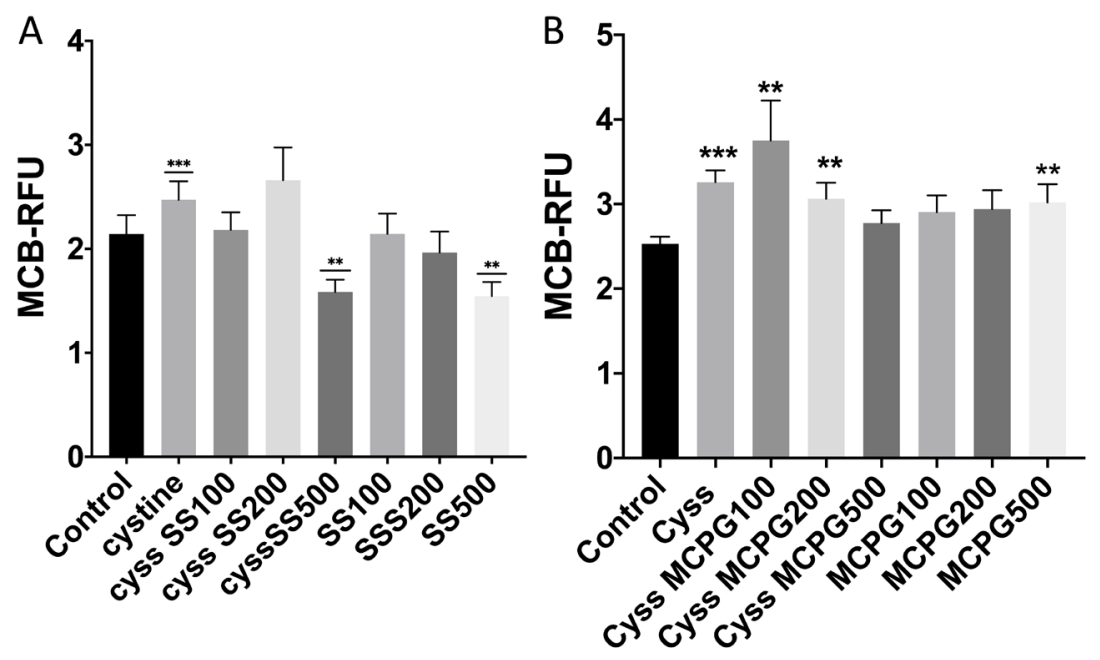

C

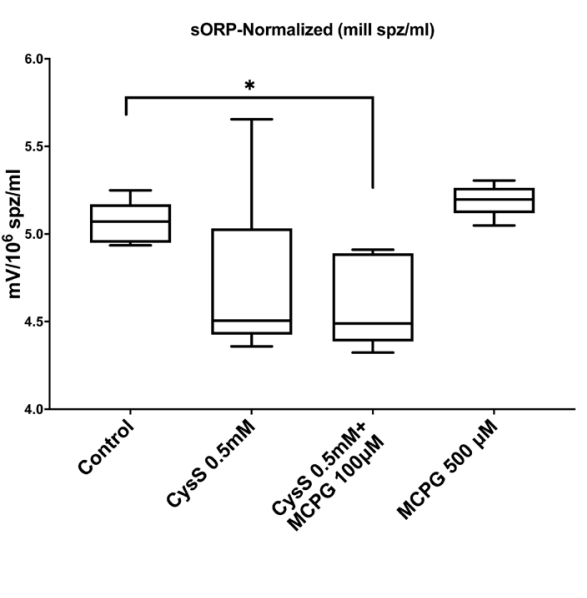

d

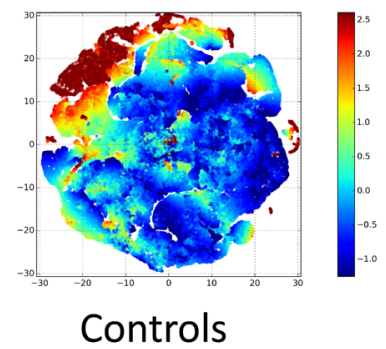

e

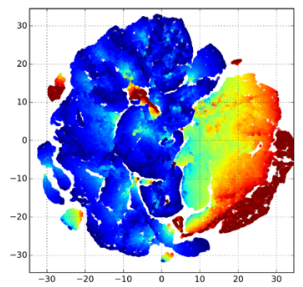

Cyss f

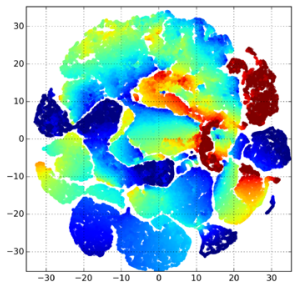

Cyss-mCPG 100

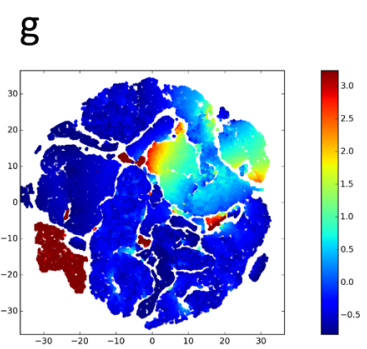

SS 500

Figure 5 Sulfasalazine reduces intracellular GSH. Stallion spermatozoa were incubated in the presence of SS and/or Cyys and mCPG as described in the 'Materials and methods' section. SS caused a marked drop in GSH content at $500 \mu \mathrm{M}$ even in the presence of Cyss $(P<0.01)$ (A). The supplementation with mCPG caused increases in GSH $(P<0.01)$ that were even bigger in the presence of $0.5 \mathrm{mM} C y s \mathrm{~s}(P<0.001)(\mathrm{B})$. The combination of $0.5 \mathrm{mM}$ Cyss and $100 \mu \mathrm{M} \mathrm{mCPG}$ also reduced the steady state redox potential (Eh) (C). A t-SNE map shows the increase in GSH content in samples supplemented with only Cyss (e), mCPG and Cyss (f), evidenced by changes in the heat map overlay (more yellowgreen-red areas) when compared with controls (d) (predominance of blue areas) and samples supplemented with SS $500 \mu \mathrm{M}$ (g) (predominance of blue areas). The color code for the heat map is depicted at the right of every t-SNE plot. Data are means \pm S.E.M. ${ }^{* * * P}<0.001 .{ }^{* * P}<0.01$; $* P<0.05$.

synergistic effect in the presence of RSL3 $(P<0.001)$ (Fig. 7C). Sulfasalazine increased VCL $(P<0.01)$, and liproxstatin-1 was able to revert the effect of SS. The straight line and average velocities showed the same behavior as that of the VCL (Fig. 7D and E). We also studied the effect on BCF; once again, both inducers of ferroptosis reduced this parameter (Fig. 7F) $P<0.001$ for erastin and $P<0.05$ for RSL3. Liproxstatin- 1 reverted the effect of RSL3 but not of erastin (Fig. 7F).

\section{Inhibition of glutamate dehydrogenase (GDH1) increases intracellular GSH and improves mitochondrial function and motility}

The increase in GSH after inhibition of the antiporter with mCPG may be explained by increased availability for intracellular glutamate that can be used for GSH synthesis. Alternatively, glutamate can be transformed into $\alpha$-ketoglutarate through glutamate dehydrogenase 1 (GDH1) and incorporated into de TCA cycle, providing reducing equivalents to recycle GSSG. This enzyme has been detected in proteomic studies in our laboratory and confirmed using western blotting and immunofluorescence (Fig. 8-1). The expression of $\mathrm{GDH} 1$, as expected, was restricted to the mid piece (Fig. 8-1). If GDH1 is inhibited, glutamate cannot be transformed into $\alpha$-ketoglutarate and intracellular concentrations will increase. This approach mimics the effect of SLC7A11 inhibition, resulting in increased intracellular glutamate. In order to test this hypothesis, we performed a series of experiments. Stallion spermatozoa were incubated in the presence of the specific inhibitor of GDH1; R162 $(20-80 \mu \mathrm{M})$, and after three and $6 \mathrm{~h}$ of incubation at $37^{\circ} \mathrm{C}$, intracellular GSH and mitochondrial activity $(\Delta \Psi \mathrm{m})$ were evaluated. Inhibition of GDH1 resulted in an increase of $>50 \%$ of the initial levels of GSH after $3 \mathrm{~h}$ of incubation $(P<0.01)$ (Fig. 8-2A). The percentage of spermatozoa showing high $\Delta \Psi \mathrm{m}$ increased after 3 and $6 \mathrm{~h}$ of incubation (Fig. $8-2 \mathrm{C}$ and $\mathrm{D})$; from $68.1 \pm 5.4$ in controls to $76.5 \pm 6.5$ 

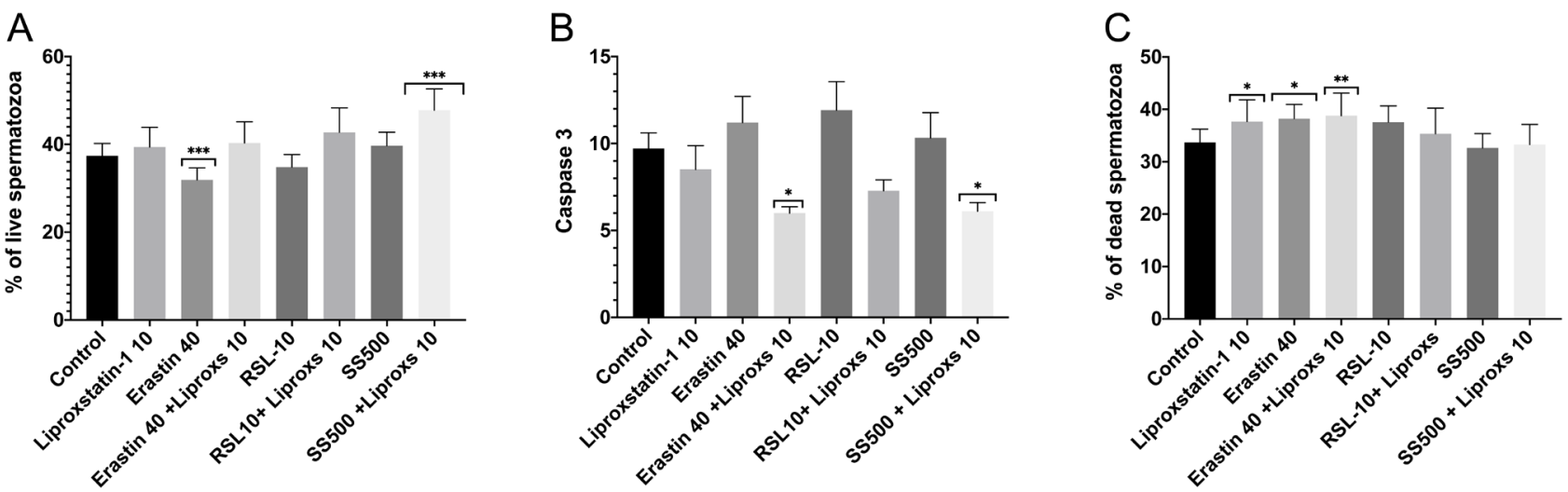

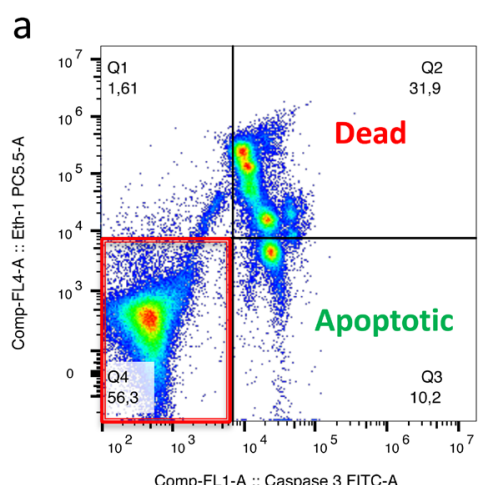

Control b

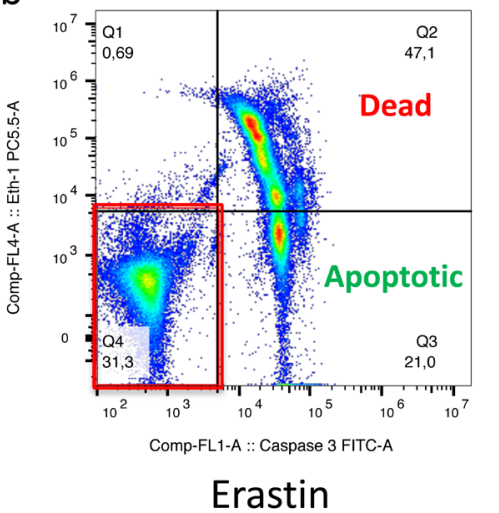

C

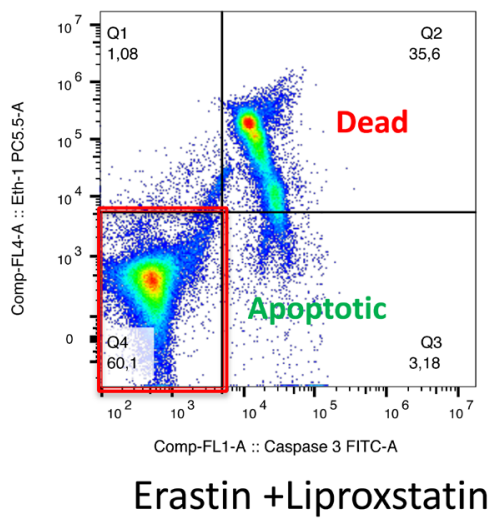

Figure 6 Effect of two different inductors of ferroptosis in stallion sperm viability. Stallion spermatozoa were incubated for $3 \mathrm{~h}$ at $37^{\circ} \mathrm{C}$ and at the final of the incubation period viability was assessed using flow cytometry. Panel A shows sperm viability, B) Caspase 3 and C) dead spermatozoa.Split samples from the same ejaculate were incubated in the presence of the inductors of ferroptosis erastin $40 \mu \mathrm{M}$ (b) and RSL3 $10 \mu \mathrm{M}$, and the inhibitor of ferroptosis liproxstatin-1 $10 \mu \mathrm{M}$ (b) compared with controls (a). Erastin caused a significant reduction of the percentage of viable spermatozoa that was prevented in presence of liproxstatin-1. Data are means \pm S.E.M. ${ }^{* * *} P<0.001 ;{ }^{* *} P<0.01 ;{ }^{*} P<0.05$.

in samples treated with $20 \mu \mathrm{M}$ R162 $(P<0.001)$ and to $76.4 \pm 5.6$ in samples treated with $40 \mu \mathrm{M}$ R162 $(P<0.01)$ after $3 \mathrm{~h}$ of incubation (Fig. 8C). After $6 \mathrm{~h}$ of incubation the percentage of spermatozoa showing high $\Delta \Psi \mathrm{m}$ increased from $64.7 \pm 7.8$ in controls to $71.5 \pm 4.8$ in samples treated with $80 \mu \mathrm{M}$ R162 $(P<0.05)$ (Fig. 8-2D). Also, mitochondrial activity increased both after three $(P<0.05)$ (Fig. 8-2E) and $6 \mathrm{~h}(P<0.01)$ (Fig. 8-2F) of incubation at $37^{\circ} \mathrm{C}$. Concomitantly due to increased intracellular GSH, the steady state oxidation potential was reduced in samples supplemented with the GDH1 inhibitor $(P<0.05)$ (Fig. 8-2b). The GDH1 inhibitor also caused increases in the percentage of motile spermatozoa (Fig. 8-3A) $(P<0.05)$ and sperm velocity (Fig. 8-3C and E). $(P<0.05)$.

\section{Metabolomics inhibition of GDH1 increases succinate, fumarate, and malate}

Inhibition of GDH1, thus blocking the transformation of glutamate into $\alpha$-ketoglutarate, caused an unexpected increase in the levels of succinate (Fig. 9-1) and of fumarate. This finding suggests that an alternative route for the formation of succinate and fumarate from glutamate is present in the stallion spermatozoa.

\section{Effect $\alpha$-ketoglutarate on stallion sperm function}

To determine the importance of $\alpha$-ketoglutarate on sperm function stallion spermatozoa were incubated in the presence of the product of GDH1, dimethyl- $\alpha$ ketoglutarate (a cell permeable form of $\alpha$ ketoglutarate) $(0 \mu \mathrm{M}, 20 \mu \mathrm{M}, 100 \mu \mathrm{M}$ and $0.5 \mu \mathrm{mM})$. After $3 \mathrm{~h}$ of incubation at $37^{\circ} \mathrm{C}$ mitochondrial function $(\Delta \Psi \mathrm{m})$, the percentage of live cells and GSH content were measured. Supplementation with dimethyl- $\alpha$ ketoglutarate did not modify the relative amount of GSH, percentage of live spermatozoa (Fig. 9-2C), or the percentage of spermatozoa showing high mitochondrial activity (Fig. 9-2B).

\section{Discussion}

In the present study, we evaluated the relationship between the SLC7A11 antiporter and mitochondrial function in the stallion spermatozoa. The major findings 
A

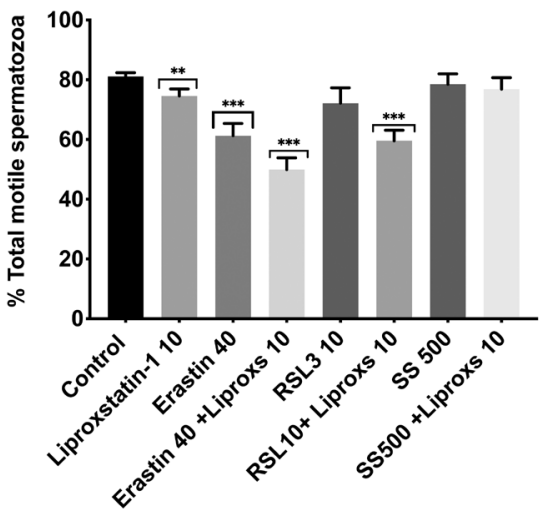

D

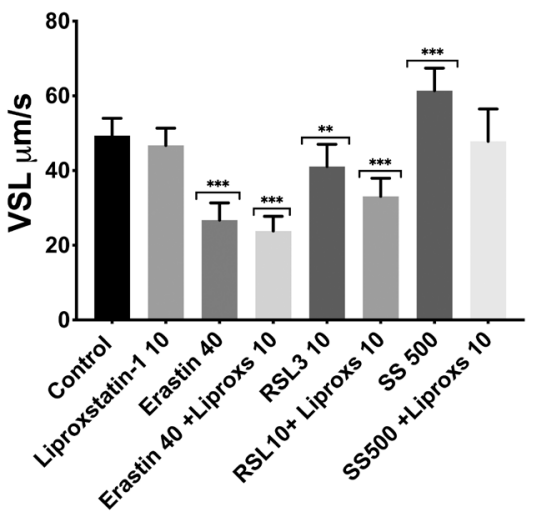

B
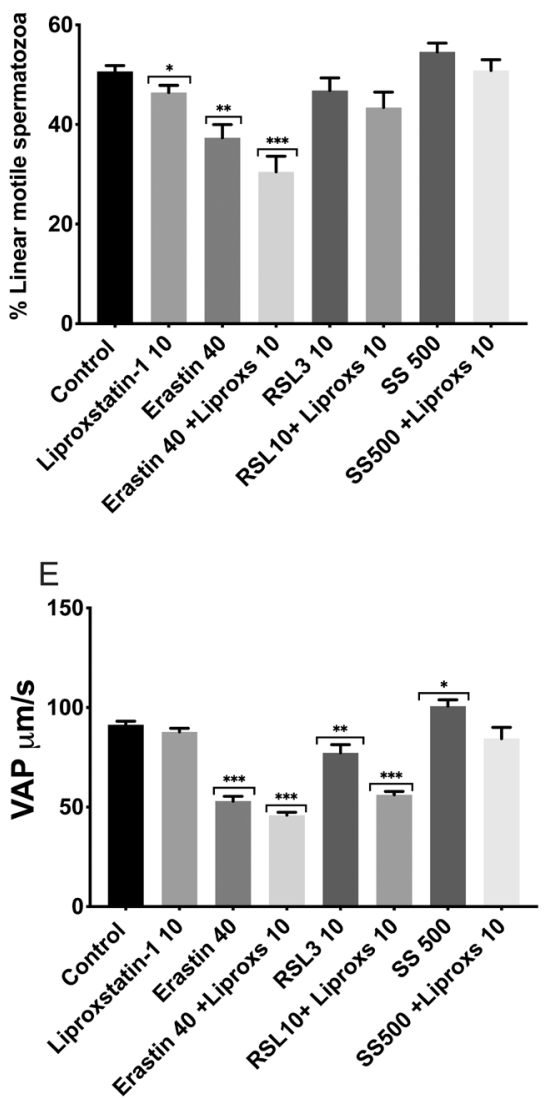

C

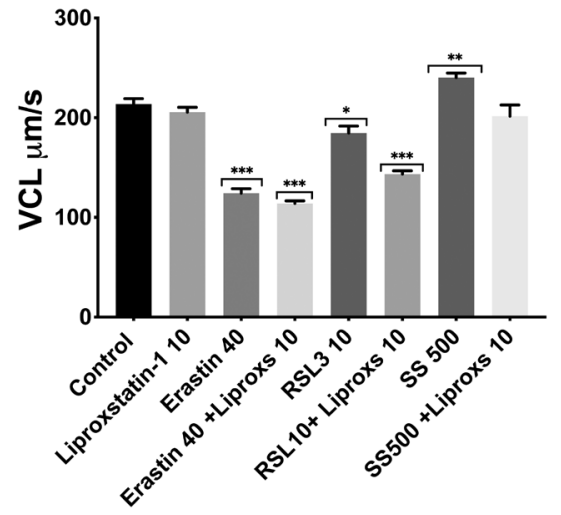

F

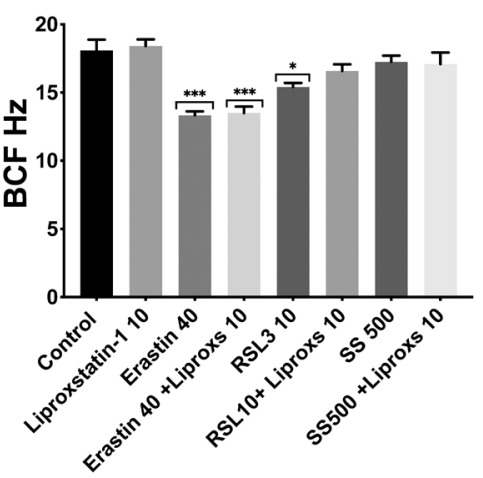

Figure 7 Effect of two different inductors of ferroptosis in stallion sperm motility, velocities and beat cross frequency (BCF). Stallion spermatozoa were incubated for $3 \mathrm{~h}$ at $37^{\circ} \mathrm{C}$ and at the final of the incubation period motility was assessed using computer-assisted sperm analysis (CASA). Split samples from the same ejaculate were incubated in the presence of the inductors of ferroptosis erastin $40 \mu \mathrm{M}$ and RSL3 $10 \mu \mathrm{M}$, and the inhibitor of ferroptosis liproxstatin-1 $10 \mu \mathrm{M}$. Erastin reduced sperm motility $(\mathrm{A})$ and $(\mathrm{B})$. Erastin and RSL3 reduced velocities (C, D and E). BCF was reduced by erastin and RSL3, the reduction induced by RSL3 was prevented by liproxstatin-1 (F). Data are means \pm S.E.M. $* * * P<0.001$; $* * P<0.01 ; * P<0.05$.

of this study are summarized in the diagram provided in Fig. 10. First, we provide additional evidence that this antiporter participates in redox regulation in spermatozoa, in addition to presenting findings suggesting that it may also have an important role in glutamate metabolism. We showed that supplementation with Cyss increases intracellular $\mathrm{GSH}$, and this increase is inhibited in the presence of BSO. This finding is evidence of the incorporation of Cyss, that after reduction to Cys is used for GSH synthesis in the spermatozoa. The findings reported here underline the importance of GSH for proper mitochondrial function in these particular cells. Inhibition of the SLC7A11 antiporter with SS caused a dramatic decrease in the percentage of spermatozoa with high mitochondrial membrane potential. This effect was even more evident with the simultaneous presence of Cyss. Changes described in the percentages of mitochondrial membrane potential in samples incubated in the presence of SS were accompanied by equivalent reductions in the percentage of live spermatozoa and intracellular glutathione. The impact of reduced availability of GSH in mitochondrial function is expected; GSH is synthetized only in the cytosol and then transported to the mitochondria were mitochondrial $\mathrm{GSH}(\mathrm{mGSH})$ is present at concentrations similar to those in the cytosol (10-14 mM) (Lluis et al. 2005, Mari et al. 2009, 2013). Although reported concentrations of GSH in spermatozoa in most domestic species is low, around $0.3 \mathrm{mM}$ (Li 1975), concentrations reported in stallions are estimated in the range of $30 \mathrm{mM}$ (Ortega-Ferrusola et al. 2019). Since mitochondria are the primary intracellular site of oxygen consumption and thus, the major source of intracellular ROS, mGSH is essential to balance the activity of Mn-SOD (the mitochondrial form of SOD) and the GSH redox node, to maintain hydrogen peroxide within physiological limits. Metabolism of $\mathrm{H}_{2} \mathrm{O}_{2}$ in the mitochondria depends on mGSH with the participation of GSH peroxidases and peroxiredoxins (Mari et al. 2009, 2013). Reduced GSH may be due to increased ROS production and/or to reduced activity 
(1)

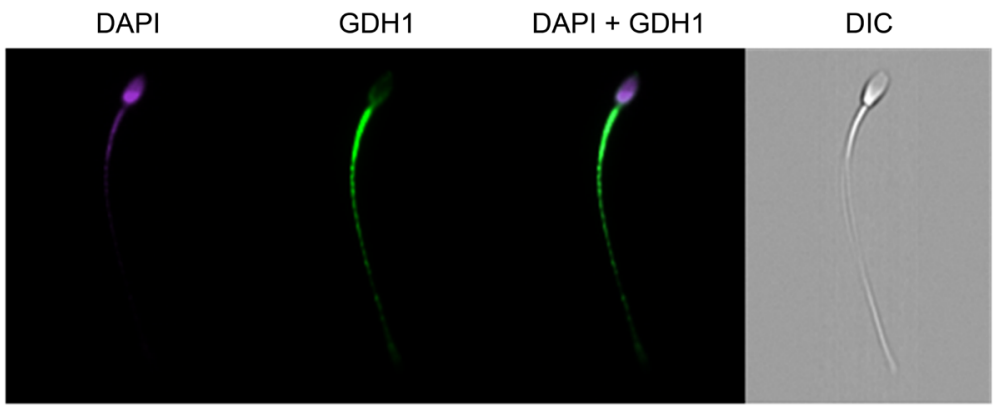

(2)

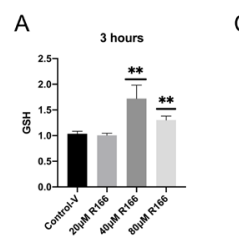

C

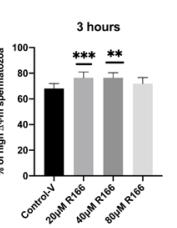

E
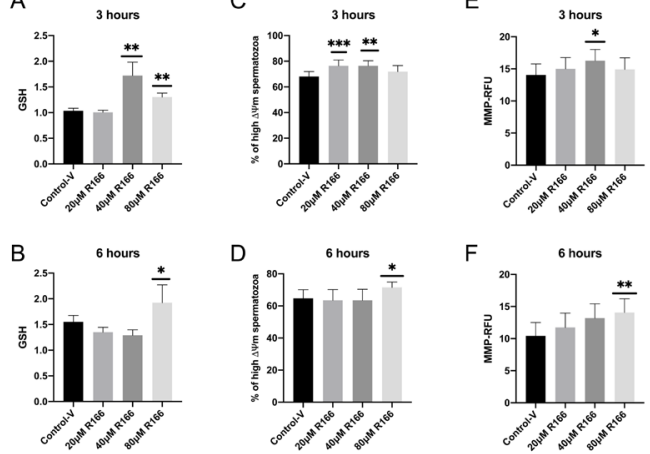

Glutamate dehydrogenase 1
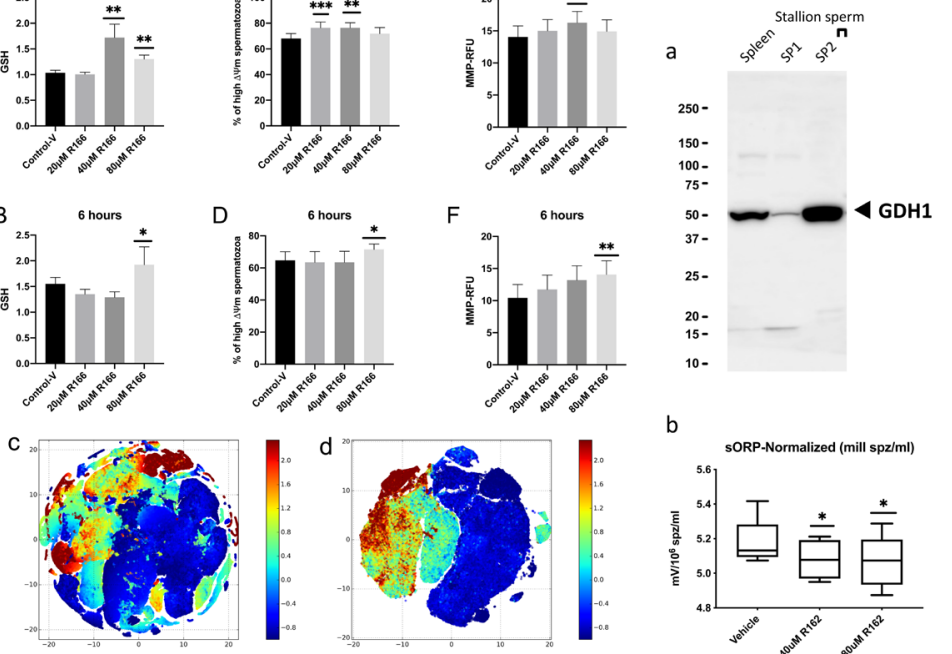

(3)

A

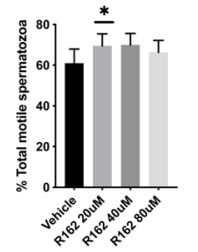

B

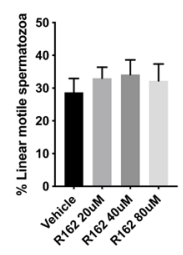

C

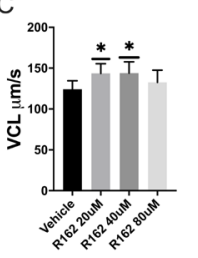

D

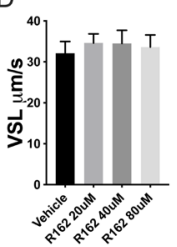

E

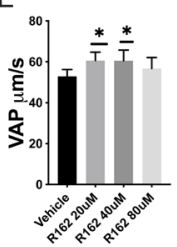

Figure 8 (1) Glutamate dehydrogenase $1(\mathrm{GDH} 1)$ is present in stallion spermatozoa. Stallion spermatozoa were processed and stained as described in material and methods and the presence and distribution of the enzyme was assessed using image flow cytometry. GDH1 was present in the mid piece. (2) Inhibition of glutamate dehydrogenase 1 (GDH1) increases intracellular GSH. Stallion spermatozoa were incubated in presence of the specific GDH1 inhibitor R166 and intracellular GSH and the mitochondrial function evaluated after 3 and 6 h of incubation at $37^{\circ} \mathrm{C}$. (A) Inhibition of GDH1 increased GSH content after 3 and after $6 \mathrm{~h}$ of incubation (B). (C and D) Inhibition of GDH1 increased the percentage of spermatozoa with high mitochondrial membrane potential after $3(\mathrm{C})$ and $6 \mathrm{~h}$ of incubation (D). (E and F) Also inhibition of GDH1 increased mitochondrial activity. The presence of Glutamate dehydrogenase 1 was also confirmed using Western blotting (a). R162 also reduced the steady state redox potential (Eh) (b). c-d tSNE maps showing changes in GSH in controls (c) and R166 treated samples. Data are means \pm S.E.M. ${ }^{* *} P<0.001 ;{ }^{* *} P<0.01 ;{ }^{*} P<0.05$. (3) Inhibition of glutamate dehydrogenase $1(\mathrm{GDH} 1)$ increases sperm motility and velocity. Stallion spermatozoa were incubated in the presence of the GDH1 inhibitor R166 during $3 \mathrm{~h}$ at $37^{\circ} \mathrm{C}$ and after the incubation period sperm motility $(\%$ of motile spermatozoa) (A) and velocities (VCL $\mu \mathrm{m} / \mathrm{s}, \mathrm{VAP} \mu \mathrm{m} / \mathrm{s}$ and VSL $\mu \mathrm{m} / \mathrm{s}$ ) (B) were measured using CASA analysis. Increases in motility VCL and VAP were detected in samples incubated in the presence of GDH1. Data are means \pm S.E.M. ${ }^{*} P<0.05$. 

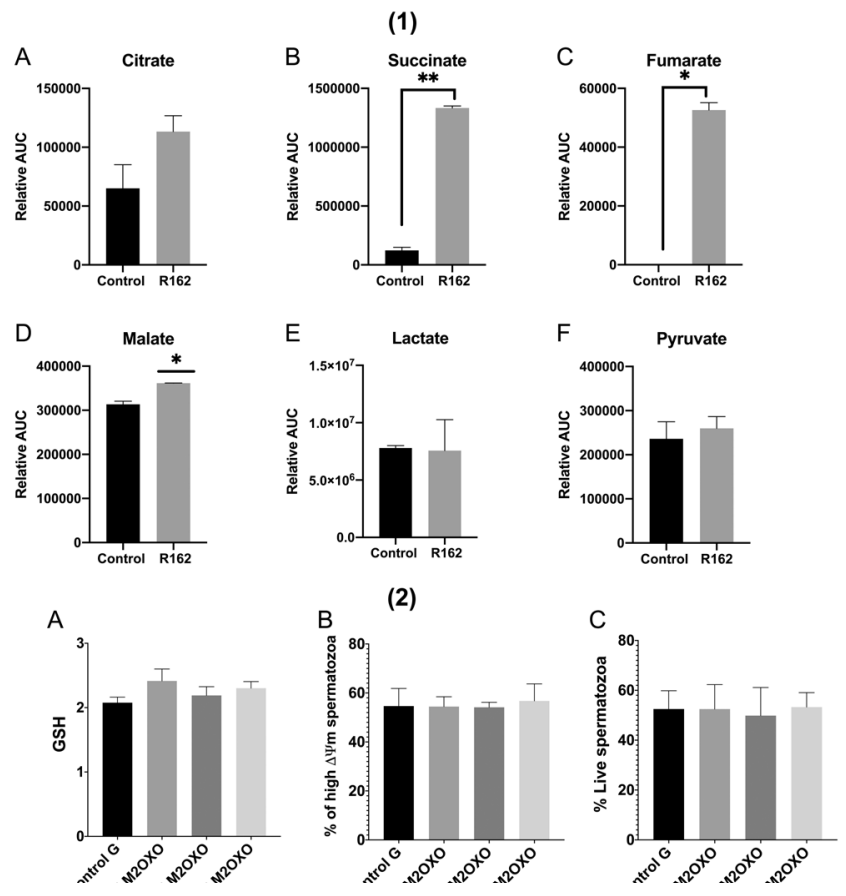

Figure 9 (1) Inhibition of glutamate dehydrogenase 1 (GDH1) modifies the stallion sperm metabolome. A) Citrate B) Succinate C) Fumarate D) Malate E) Lactate F) Pyruvate. Succinate and fumarate increased after GDH1 inhibition indicating that glutamate may be metabolized, even more efficiently, through pathways alternative to glutamate dehydrogenase. (2) Effect of supplementation with dimethyl $\alpha$-ketoglutarate for $3 \mathrm{~h}$ at $37^{\circ} \mathrm{C}$ on GSH content, mitochondrial membrane potential and viability of stallion spermatozoa. A) GSH B) High Mitochondrial Membrane Potential C) Viability. Data are means \pm S.E.M. ${ }^{* *} P<0.01 ;{ }^{*} P<0.05$.

of the SLC7A11 antiporter and depletion of glutathione peroxidase 4 (GPX4) (Yu et al. 2019). This induces a form of programed cell death termed ferroptosis, that has been reported in the male germ line (Bromfield et al. 2019). GPX-4 forms the mitochondrial sheath of the spermatozoa, being crucial for the maintenance of sperm functionality (Brigelius-Flohe \& Flohe 2019). To test the possibility that a ferroptotic mechanism is activated after SLC7A11 inhibition, we performed experiments comparing the effect of SS with known inducers of ferroptosis, such as erastin and RSL3. Erastin reduced the percentage of live spermatozoa, an effect that was combated by the ferroptosis inhibitor liproxstatin-1. This finding supports the existence of ferroptosis in ejaculated stallion spermatozoa. Interestingly, the simultaneous presence of SS and liproxtatin-1 resulted in increased viability and reduced expression of caspase 3, suggesting different mechanisms of cell death caused by erastin and sulfasalazine. However, although erastin and RSL3 reduced motility and velocities in spermatozoa, their effects were not prevented by liproxstatin-1. We also investigated effects on BCF, that is related to increased sperm functionality and ATP content (Mannowetz et al. 2012). This parameter was reduced in the presence of

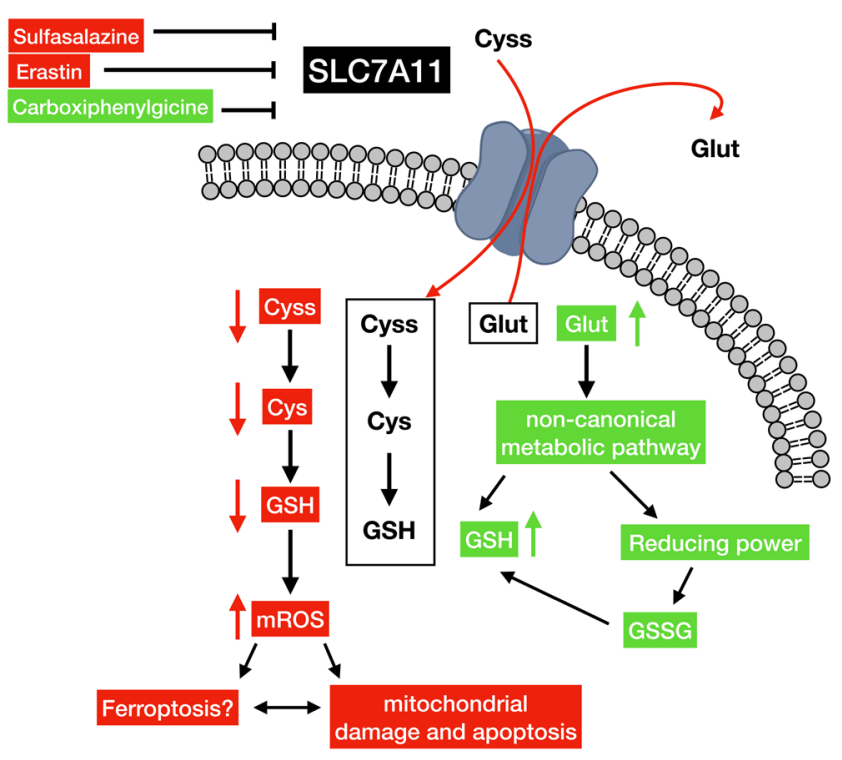

Figure 10 Schematic representation of the major hypothesis and findings of this study. The SLC7A11 antiporter contributes to redox regulation in stallion spermatozoa through the exchange of extracelular cystine (Cyss) for intracelullar glutamate (Glut). Cyss intracellularly is reduced to Cys that is used for GSH synthesis. Inhibition of this antiporter may lead to two major situations. In red, inhibition of the SLC7A11 antiporter with sulfasalazine leads to reduced intracellular Cys and then reduction in intracellular GSH. This causes redox deregulation and mitochondrial damage. Erastin also causes inhibition of the SLC7A11 antiporter. The deregulation of redox homeostasis may lead to increase of lipoperoxides (LPO) and the induction of ferroptosis. Alternatively (in green), the use of a much more potent inhibitor of the antiporter, may lead to greater increases of intracellular glutamate that may be metabolized following an alternative pathway. This alternative pathway produces reducing power to recycle GSSG to GSH and maintain redox homeostasis and mitochondrial function.

both inductors of ferroptosis, but liproxstatin-1 only reverted the effect of RSL3. The differential effect on viability and motility of erastin could relate to the effect of this drug on the voltage-dependent anion channel in the mitochondria (VDAC) that regulates the metabolic flux across the outer mitochondrial membrane (BlachlyDyson \& Forte 2001). Overall these results may indicate that different forms of cellular demise activated under redox deregulation may be present in ejaculated spermatozoa. However, further research is warranted to fully characterize these mechanisms and the crosstalk between them. It is also worth noting that the best spermatozoa from the ejaculate were selected through colloidal centrifugation; different responses in different sperm subpopulations ought to be considered.

The more potent inhibitor methyl S-4carboxyphenylglycine (mCPG), increased the percentage of spermatozoa with high mitochondrial membrane potential $(\Delta \Psi \mathrm{m})$ when Cyss was also present. Incubation in the presence of $\mathrm{mCPG}$ resulted in reduced percentages of live spermatozoa that was prevented in 
the presence of Cyss. The percentage of spermatozoa with high mitochondrial membrane potential and intracellular GSH increased when Cyss was also present, and in this case, without impact on sperm viability. The improvement in $\Delta \Psi \mathrm{m}$ was in parallel with increased intracellular GSH and thus can be explained through this mechanism.

It is paradoxical that both increases or decreases in GSH may occur using different inhibitors of the SLC7A11 antiporter. One possible explanation may relate to the dual effects of the antiporter. This is, as a regulator of the availability of Cys for GSH synthesis and that it may also participate in the regulation of glutamate metabolism (Shin et al. 2017). The mCPG is a very potent inhibitor of the SLC7A11 antiporter, rapidly leading to increased intracellular glutamate accumulation (Patel et al. 2004). In this model, SLC7A11 inhibition blocks the exchange of intracellular glutamate for extracellular Cyss that leads to increased intracellular glutamate, an effect that may be enhanced in the presence of extracelullar Cyss. After transformation to $\alpha$-ketoglutarate, glutamate may feed the mitochondrial TCA cycle (Jin et al. 2015). In fact, glutamate, supports the production of molecules such as GSH and NDPH after transformation into $\alpha$-ketoglutarate by the enzyme glutamate dehydrogenase 1 (GDH1) (Wise \& Thompson 2010, Jin et al. 2015). This mechanism provides a plausible explanation for our findings. Further supporting this hypothesis antioxidant effects of $\alpha$-ketoglutarate supplementation have been previously reported in spermatozoa (Li et al. 2010). Recent reports in rabbit spermatozoa, showing increased GSH after glutamine supplementation also support this hypothesis (Zhu et al. 2017). To increase intracellular glutamate, we blocked the conversion of glutamate to $\alpha$-ketoglutarate through the inhibition of the glutamate dehydrogenase 1 (GDH1) enzyme. Inhibition of $\mathrm{GDH} 1$, blocking the conversion of glutamate into $\alpha$-ketoglutarate and incorporation into the TCA cycle, caused increased mitochondrial membrane potential and intracellular GSH (Fig. 8). This may indicate that excess glutamate is not necessarily metabolized through the GDH1 pathway, and that alternative routes are present in stallion spermatozoa. Alternative routes have been reported in tumoral cells (DeBerardinis \& Cheng 2010, Son et al. 2013, Boroughs \& DeBerardinis 2015). Whereas in most cells GDH1 converts glutamate into $\alpha$-ketoglutarate, some tumors use a distinct pathway involving transaminases (GOT1), in which glutamate is converted to pyruvate increasing the NADPH/NADP ${ }^{+}$ ratio and maintaining the cellular redox state (Son et al. 2013). A similar mechanism has recently been described. Under impaired mitochondrial metabolism cytosolic reductive carboxylation of glutamine regenerates NADH via malate dehydrogenase (Gaude et al. 2018). This is a very similar metabolic landscape to the one observed in our study in stallion spermatozoa after GDH1 inhibition. Interestingly transaminase activity has been reported in mammalian spermatozoa (Flipse 1960). We conducted metabolomic studies using UHPLC/MS/MS after inhibition of GDH1, focusing on metabolites of the TCA cycle. Treatment with R162, the specific GDH1 inhibitor, caused a dramatic increase in succinate, that was unexpected, since succinate is produced downstream of $\alpha$-ketoglutarate in the TCA cycle. There was also a dramatic increase in fumarate in R162 treated samples. This observation suggests, as indicated above, that glutamate can also be metabolized through alternative pathways, probably by transaminases (DeBerardinis et al. 2007). In this model, the secretion of amino groups from glutamate is used to generate NADPH. Fumarate is also produced in the process of ammonia detoxification, through the cleavage of arginine succinate to form fumarate and arginine. Fumarate returns to the citric acid cycle (Shambaugh 1977). This mechanism may explain our findings since glutamate can be metabolized to arginine (Yelamanchi et al. 2016). Moreover, and further supporting this hypothesis a functional urea cycle has been described in mammalian spermatozoa (Dietz \& Flipse 1969). As previously mentioned, transaminase activity has been reported in mammalian spermatozoa (Flipse 1960), and also in a recent proteomic study from our laboratory (Martin-Cano et al. 2020). These findings may indicate that there is a non-canonical pathway for glutamate metabolism, producing reducing power to recycle GSSG into GSH. This provides an explanation for our findings of increased GSH and mitochondrial membrane potential after $\mathrm{GDH} 1$ inhibition. A similar pathway has been described in pancreatic ductal adenocarcinoma cells (PADC) in which glutamate in the cytoplasm forms aspartate and then oxaloacetate that is converted to malate and then pyruvate increasing the NADPH/ NAD+ ratio and in this way maintains cellular redox (Son et al. 2013). Increased malate was also observed in our study after GDH-1 inhibition. In this model, dimethyl $\alpha$-ketoglutarate does not restore cell growth after glutamate deprivation, indicating a glutamate metabolism different from canonical models. As in the aforementioned study and in ours, supplementation with dimethyl $\alpha$-ketoglutarate did not improve stallion sperm function, also suggesting alternative glutamate metabolic pathways in spermatozoa. This provides a plausible explanation to our findings of improved sperm function after GDH1 inhibition, and could also provide an explanation to increased GSH after SLC7A11 inhibition. Interestingly, for the first time, our findings suggest, that the stallion spermatozoa show an important metabolic plasticity. This is an interesting finding that could open new routes for the development of novel strategies for the management of male factor infertility, and development of new strategies for sperm conservation.

In conclusion, the results reported here describe the importance of the activity of the SLC7A11 antiporter for 
proper mitochondrial function in stallion spermatozoa, providing Cys for GSH synthesis (Martensson et al. 1990). They also suggest that different forms of programmed cell death may be present in the spermatozoa that warrant further research. Our findings provide evidence of a high plasticity in the metabolism of the spermatozoa and in the regulation of their redox potential. In addition, an active and complex glutamate metabolism may be present in the stallion spermatozoa (Dietz \& Flipse 1967, 1969, Flipse \& Dietz 1969, Flipse et al. 1969, DeBerardinis et al. 2007, Jin et al. 2015, Yelamanchi et al. 2016) that also warrants further research. The findings reported here are in line with the growing body of scientific evidence indicating that mitochondria are crucial for stallion sperm functionality and fertility, being a hallmark of fertile spermatozoa (Gibb et al. 2014, Davila et al. 2016).

\section{Declaration of interest}

The authors declare that there is no conflict of interest that could be perceived as prejudicing the impartiality of the research reported.

\section{Funding}

The authors received financial support for this study from the Ministerio de Economía y Competitividad-FEDER, Madrid, Spain, through grants AGL2017-83149-R and PID2019107797RA-I00 and Junta de Extremadura-FEDER (IB16030 and GR18008 and PD 18005), J M O R holds a Predoctoral grant from Junta de Extremadura-FEDER (PD 18005), G G P holds a PhD grant from the Ministry of Science, Madrid, Spain (PRE 2018-083354).

\section{Author contribution statement}

J M O-R, FE M-C, A S, M C G and C O-F performed experiments, contributed to data analysis. G G-P performed experiments and writing edition (native English speaker). J A T, E R and J M performed experiments. F J P conceived the study, involved in supervision, data analysis and interpretation, writing and funding acquisition.

\section{References}

Amaral A, Castillo J, Estanyol JM, Ballesca JL, Ramalho-Santos J \& Oliva R 2013 Human sperm tail proteome suggests new endogenous metabolic pathways. Molecular and Cellular Proteomics 12 330-342. (https://doi. org/10.1074/mcp.M112.020552)

Amaral A, Paiva C, Attardo Parrinello C, Estanyol JM, Ballesca JL, Ramalho-Santos J \& Oliva R 2014 Identification of proteins involved in human sperm motility using high-throughput differential proteomics. Journal of Proteome Research 13 5670-5684. (https://doi.org/10.1021/ pr500652y)

Aparicio IM, Espino J, Bejarano I, Gallardo-Soler A, Campo ML, Salido GM, Pariente JA, Pena FJ \& Tapia JA 2016 Autophagy-related proteins are functionally active in human spermatozoa and may be involved in the regulation of cell survival and motility. Scientific Reports 6 33647. (https://doi.org/10.1038/srep33647)

Balao da Silva CM, Ortega Ferrusola C, Gallardo Bolanos JM, Plaza Davila M, Martin-Munoz P, Morrell JM, Rodriguez Martinez H \& Pena FJ 2014 Effect of overnight staining on the quality of flow cytometric sorted stallion sperm: comparison with tradtitional protocols. Reproduction in Domestic Animals 49 1021-1027. (https://doi.org/10.1111/rda.12431)

Blachly-Dyson E \& Forte M 2001 VDAC channels. IUBMB Life $\mathbf{5 2}$ 113-118. (https://doi.org/10.1080/15216540152845902)

Boroughs LK \& DeBerardinis RJ 2015 Metabolic pathways promoting cancer cell survival and growth. Nature Cell Biology 17 351-359. (https://doi.org/10.1038/ncb3124)

Brigelius-Flohe R \& Flohe L 2020 Regulatory phenomena in the glutathione peroxidase superfamily. Antioxidants and Redox Signaling 33 498-516. (https://doi.org/10.1089/ars.2019.7905)

Bromfield EG, Walters JLH, Cafe SL, Bernstein IR, Stanger SJ, Anderson AL, Aitken RJ, McLaughlin EA, Dun MD, Gadella BM et al.2019 Differential cell death decisions in the testis: evidence for an exclusive window of ferroptosis in round spermatids. Molecular Human Reproduction 25 241-256. (https://doi.org/10.1093/molehr/gaz015)

Capek J, Hauschke M, Bruckova L \& Rousar T 2017 Comparison of glutathione levels measured using optimized monochlorobimane assay with those from ortho-phthalaldehyde assay in intact cells. Journal of Pharmacological and Toxicological Methods 88 40-45. (https://doi. org/10.1016/j.vascn.2017.06.001)

Circu ML, Rodriguez C, Maloney R, Moyer MP \& Aw TY 2008 Contribution of mitochondrial GSH transport to matrix GSH status and colonic epithelial cell apoptosis. Free Radical Biology and Medicine $\mathbf{4 4}$ 768-778. (https://doi.org/10.1016/j.freeradbiomed.2007.09.011)

Coppola S \& Ghibelli L 2000 GSH extrusion and and the mitochondrial pathway of apoptotic signalling. Biochemical Society Transactions $\mathbf{2 8}$ 56-61. (https://doi.org/10.1042/bst0280056)

Darr CR, Varner DD, Teague S, Cortopassi GA, Datta S \& Meyers SA 2016 Lactate and pyruvate are major sources of energy for stallion sperm with dose effects on mitochondrial function, motility, and ROS production. Biology of Reproduction 95 34. (https://doi.org/10.1095/ biolreprod.116.140707)

Davila MP, Munoz PM, Bolanos JM, Stout TA, Gadella BM, Tapia JA, da Silva CB, Ferrusola CO \& Pena FJ 2016 Mitochondrial ATP is required for the maintenance of membrane integrity in stallion spermatozoa, whereas motility requires both glycolysis and oxidative phosphorylation. Reproduction 152 683-694. (https://doi.org/10.1530/REP-16-0409)

DeBerardinis RJ \& Cheng T 2010 Q's next: the diverse functions of glutamine in metabolism, cell biology and cancer. Oncogene 29 313-324. (https://doi.org/10.1038/onc.2009.358)

DeBerardinis RJ, Mancuso A, Daikhin E, Nissim I, Yudkoff M, Wehrli S \& Thompson CB 2007 Beyond aerobic glycolysis: transformed cells can engage in glutamine metabolism that exceeds the requirement for protein and nucleotide synthesis. PNAS 104 19345-19350. (https://doi. org/10.1073/pnas.0709747104)

Dietz RW \& Flipse RJ 1967 Metabolism of bovine semen. XVI. Pathways for arginine utilization. Journal of Dairy Science 50 1973-1977. (https:// doi.org/10.3168/jds.S0022-0302(67)87755-5)

Dietz RW \& Flipse RJ 1969 Metabolism of bovine semen. XX. Role of ammonia in interactions between the citric acid and urea cycles. Biology of Reproduction 1 200-206. (https://doi.org/10.1095/biolreprod1.2.200)

Flipse RJ 1960 Metabolism of bovine semen. IX. Glutamic-oxalacetic and glutamic-pyruvic transaminase activities. Journal of Dairy Science $\mathbf{4 3}$ 773-776. (https://doi.org/10.3168/jds.S0022-0302(60)90237-X)

Flipse RJ \& Dietz RW 1969 Metabolism of bovine semen. XVII. Oxidative metabolism of glutamate. Journal of Dairy Science 52 113-116. (https:// doi.org/10.3168/jds.S0022-0302(69)86511-2)

Flipse RJ, Sexton TJ \& Dietz RW 1969 Metabolism of bovine semen. 18. Effect of keto acids on glutamate metabolism. Journal of Dairy Science 52 386-389. (https://doi.org/10.3168/jds.s0022-0302(69)86565-3)

Gallardo Bolanos JM, Balao da Silva CM, Martin Munoz P, Morillo Rodriguez A, Plaza Davila M, Rodriguez-Martinez H, Aparicio IM, Tapia JA, Ortega Ferrusola C \& Peña FJ 2014 Phosphorylated AKT preserves stallion sperm viability and motility by inhibiting caspases 3 and 7. Reproduction 148 221-235. (https://doi.org/10.1530/ REP-13-0191) 
Gaude E, Schmidt C, Gammage PA, Dugourd A, Blacker T, Chew SP, SaezRodriguez J, O'Neill JS, Szabadkai G, Minczuk M et al. 2018 NADH shuttling couples cytosolic reductive carboxylation of glutamine with glycolysis in cells with mitochondrial dysfunction. Molecular Cell 69 581-593.e7. (https://doi.org/10.1016/j.molcel.2018.01.034)

Gibb Z, Lambourne SR \& Aitken RJ 2014 The paradoxical relationship between stallion fertility and oxidative stress. Biology of Reproduction 91 77. (https://doi.org/10.1095/biolreprod.114.118539)

Gibb Z, Lambourne SR, Quadrelli J, Smith ND \& Aitken RJ 2015 L-carnitine and pyruvate are prosurvival factors during the storage of stallion spermatozoa at room temperature. Biology of Reproduction 93 104. (https://doi.org/10.1095/biolreprod.115.131326)

Griffin RA, Baker M, Aitken RJ, Swegen A \& Gibb Z 2019 What makes a fertile sperm? Unique molecular attributes of stallion fertility. Reproduction 158 R125-R137. (https://doi.org/10.1530/REP-19-0060)

Jin L, Li D, Alesi GN, Fan J, Kang HB, Lu Z, Boggon TJ, Jin P, Yi H, Wright ER et al. 2015 Glutamate dehydrogenase 1 signals through antioxidant glutathione peroxidase 1 to regulate redox homeostasis and tumor growth. Cancer Cell 27 257-270. (https://doi.org/10.1016/j. ccell.2014.12.006)

Kalyanaraman B 2013 Teaching the basics of redox biology to medical and graduate students: oxidants, antioxidants and disease mechanisms. Redox Biology 1 244-257. (https://doi.org/10.1016/j. redox.2013.01.014)

Lee D, Moawad AR, Morielli T, Fernandez MC \& O'Flaherty C 2017 Peroxiredoxins prevent oxidative stress during human sperm capacitation. Molecular Human Reproduction 23 106-115. (https://doi. org/10.1093/molehr/gaw081)

Li TK 1975 The glutathione and thiol content of mammalian spermatozoa and seminal plasma. Biology of Reproduction 12 641-646. (https://doi. org/10.1095/biolreprod12.5.641)

Li SF, Liu HX, Zhang YB, Yan YC \& Li YP 2010 The protective effects of alpha-ketoacids against oxidative stress on rat spermatozoa in vitro. Asian Journal of Andrology 12 247-256. (https://doi.org/10.1038/ aja.2009.78)

Lluis JM, Morales A, Blasco C, Colell A, Mari M, Garcia-Ruiz C \& Fernandez-Checa JC 2005 Critical role of mitochondrial glutathione in the survival of hepatocytes during hypoxia. Journal of Biological Chemistry 280 3224-3232. (https://doi.org/10.1074/jbc.M408244200)

Mannowetz N, Wandernoth PM \& Wennemuth G 2012 Glucose is a pHdependent motor for sperm beat frequency during early activation. PLOS ONE 7 e41030. (https://doi.org/10.1371/journal.pone.0041030)

Mari M, Morales A, Colell A, Garcia-Ruiz C \& Fernandez-Checa JC 2009 Mitochondrial glutathione, a key survival antioxidant. Antioxidants and Redox Signaling 11 2685-2700. (https://doi.org/10.1089/ ARS.2009.2695)

Mari M, Morales A, Colell A, Garcia-Ruiz C, Kaplowitz N \& FernandezCheca JC 2013 Mitochondrial glutathione: features, regulation and role in disease. Biochimica et Biophysica Acta 1830 3317-3328. (https://doi. org/10.1016/j.bbagen.2012.10.018)

Martensson J, Lai JC \& Meister A 1990 High-affinity transport of glutathione is part of a multicomponent system essential for mitochondrial function. PNAS 87 7185-7189. (https://doi.org/10.1073/pnas.87.18.7185)

Martin Munoz P, Ortega Ferrusola C, Vizuete G, Plaza Davila M, Rodriguez Martinez H \& Pena FJ 2015 Depletion of intracellular thiols and increased production of 4-hydroxynonenal that occur during cryopreservation of stallion spermatozoa lead to caspase activation, loss of motility, and cell death. Biology of Reproduction 93 143. (https://doi. org/10.1095/biolreprod.115.132878)

Martin-Cano FE, Gaitskell-Phillips G, Ortiz-Rodriguez JM, SilvaRodriguez A, Roman Á, Rojo-Dominguez P, Alonso-Rodriguez E, Tapia JA, Gil MC, Ortega-Ferrusola C et al. 2020 Proteomic profiling of stallion spermatozoa suggests changes in sperm metabolism and compromised redox regulation after cryopreservation. Journal of Proteomics 221 103765. (https://doi.org/10.1016/j.jprot.2020.103765)

Morrell JM, Garcia BM, Pena FJ \& Johannisson A 2011 Processing stored stallion semen doses by single layer centrifugation. Theriogenology $\mathbf{7 6}$ 1424-1432. (https://doi.org/10.1016/j.theriogenology.2011.06.011)

O'Flaherty C 2015 Redox regulation of mammalian sperm capacitation. Asian Journal of Andrology 17 583-590. (https://doi.org/10.4103/1008682X.153303)
O'Flaherty C \& Matsushita-Fournier D 2017 Reactive oxygen species and protein modifications in spermatozoa. Biology of Reproduction $\mathbf{9 7}$ 577-585. (https://doi.org/10.1093/biolre/iox104)

Ortega Ferrusola C, Gonzalez Fernandez L, Macias Garcia B, SalazarSandoval C, Morillo Rodriguez A, Rodriguez Martinez H, Tapia JA \& Pena FJ 2009 Effect of cryopreservation on nitric oxide production by stallion spermatozoa. Biology of Reproduction 81 1106-1111. (https:// doi.org/10.1095/biolreprod.109.078220)

Ortega-Ferrusola C, Macias Garcia B, Suarez Rama V, GallardoBolanos JM, Gonzalez-Fernandez L, Tapia JA, Rodriguez-Martinez H \& Pena FJ 2009 Identification of sperm subpopulations in stallion ejaculates: changes after cryopreservation and comparison with traditional statistics. Reproduction in Domestic Animals 44 419-423. (https://doi.org/10.1111/j.1439-0531.2008.01097.x)

Ortega-Ferrusola C, Anel-Lopez L, Martin-Munoz P, Ortiz-Rodriguez JM, Gil MC, Alvarez M, de Paz P, Ezquerra LJ, Masot AJ, Redondo E et al. 2017 Computational flow cytometry reveals that cryopreservation induces spermptosis but subpopulations of spermatozoa may experience capacitation-like changes. Reproduction 153 293-304. (https://doi. org/10.1530/REP-16-0539)

Ortega-Ferrusola C, Martin Munoz P, Ortiz-Rodriguez JM, AnelLopez L, Balao da Silva C, Alvarez M, de Paz P, Tapia JA, Anel L, SilvaRodriguez A et al. 2019 Depletion of thiols leads to redox deregulation, production of 4-hydroxinonenal and sperm senescence: a possible role for GSH regulation in spermatozoadagger. Biology of Reproduction 100 1090-1107. (https://doi.org/10.1093/biolre/ioy241)

Ortiz-Rodriguez JM, Balao da Silva C, Masot J, Redondo E, Gazquez A, Tapia JA, Gil C, Ortega-Ferrusola C \& Pena FJ 2019a Rosiglitazone in the thawing medium improves mitochondrial function in stallion spermatozoa through regulating Akt phosphorylation and reduction of caspase 3. PLOS ONE 14 e0211994. (https://doi.org/10.1371/journal. pone.0211994)

Ortiz-Rodriguez JM, Martin-Cano FE, Ortega-Ferrusola C, Masot J, Redondo E, Gazquez A, Gil MC, Aparicio IM, Rojo-Dominguez P, Tapia JA et al. 2019b The incorporation of cystine by the soluble carrier family 7 member 11 (SLC7A11) is a component of the redox regulatory mechanism in stallion spermatozoadagger. Biology of Reproduction 101 208-222. (https://doi.org/10.1093/biolre/ioz069)

Paiva C, Amaral A, Rodriguez M, Canyellas N, Correig X, Ballesca JL, Ramalho-Santos J \& Oliva R 2015 Identification of endogenous metabolites in human sperm cells using proton nuclear magnetic resonance ((1) H-NMR) spectroscopy and gas chromatography-mass spectrometry (GC-MS). Andrology 3 496-505. (https://doi.org/10.1111/ andr.12027)

Patel SA, Warren BA, Rhoderick JF \& Bridges RJ 2004 Differentiation of substrate and non-substrate inhibitors of transport system xc(-): an obligate exchanger of L-glutamate and L-cystine. Neuropharmacology 46 273-284. (https://doi.org/10.1016/j.neuropharm.2003.08.006)

Pena FJ, O'Flaherty C, Ortiz Rodriguez JM, Martin Cano FE, GaitskellPhillips GL, Gil MC \& Ortega Ferrusola C 2019 Redox regulation and oxidative stress: the particular case of the stallion spermatozoa. Antioxidants 8 567. (https://doi.org/10.3390/antiox8110567)

Ribas V, Garcia-Ruiz C \& Fernandez-Checa JC 2014 Glutathione and mitochondria. Frontiers in Pharmacology 5 151. (https://doi.org/10.3389/ fphar.2014.00151)

Salicioni AM, Platt MD, Wertheimer EV, Arcelay E, Allaire A, Sosnik J \& Visconti PE 2007 Signalling pathways involved in sperm capacitation. Society of Reproduction and Fertility Supplement 65 245-259.

Shambaugh GE, 3rd 1977 Urea biosynthesis I. The urea cycle and relationships to the citric acid cycle. American Journal of Clinical Nutrition 30 2083-2087. (https://doi.org/10.1093/ajcn/30.12.2083)

Shekhar K, Brodin P, Davis MM \& Chakraborty AK 2014 Automatic classification of cellular expression by nonlinear stochastic embedding (ACCENSE). PNAS 111 202-207. (https://doi.org/10.1073/ pnas.1321405111)

Shin CS, Mishra P, Watrous JD, Carelli V, D'Aurelio M, Jain M \& Chan DC 2017 The glutamate/cystine xCT antiporter antagonizes glutamine metabolism and reduces nutrient flexibility. Nature Communications 8 15074. (https://doi.org/10.1038/ncomms15074)

Son J, Lyssiotis CA, Ying H, Wang X, Hua S, Ligorio M, Perera RM, Ferrone CR, Mullarky E, Shyh-Chang N et al. 2013 Glutamine supports 
pancreatic cancer growth through a KRAS-regulated metabolic pathway. Nature 496 101-105. (https://doi.org/10.1038/nature12040)

Swegen A, Curry BJ, Gibb Z, Lambourne SR, Smith ND \& Aitken RJ 2015 Investigation of the stallion sperm proteome by mass spectrometry. Reproduction 149 235-244. (https://doi.org/10.1530/REP-14-0500)

Swegen A, Lambourne SR, Aitken RJ \& Gibb Z 2016 Rosiglitazone improves stallion sperm motility, ATP content, and mitochondrial function. Biology of Reproduction 95 107. (https://doi.org/10.1095/ biolreprod.116.142687)

Tapia JA, Macias-Garcia B, Miro-Moran A, Ortega-Ferrusola C, Salido GM, Pena FJ \& Aparicio IM 2012 The membrane of the mammalian spermatozoa: much more than an inert envelope. Reproduction in Domestic Animals 47 (Supplement 3) 65-75. (https://doi.org/10.1111/ j.1439-0531.2012.02046.x)

Vidament M, Magistrini M, Le Foll Y, Levillain N, Yvon JM, Duchamp G \& Blesbois E 2012 Temperatures from 4 to 15 degrees $C$ are suitable for preserving the fertilizing capacity of stallion semen stored for $22 \mathrm{~h}$ or more in INRA96 extender. Theriogenology 78 297-307. (https://doi. org/10.1016/j.theriogenology.2012.01.018)

Wise DR \& Thompson CB 2010 Glutamine addiction: a new therapeutic target in cancer. Trends in Biochemical Sciences 35 427-433. (https:// doi.org/10.1016/j.tibs.2010.05.003)
Yelamanchi SD, Jayaram S, Thomas JK, Gundimeda S, Khan AA, Singhal A, Keshava Prasad TS, Pandey A, Somani BL \& Gowda H 2016 A pathway map of glutamate metabolism. Journal of Cell Communication and Signaling 10 69-75. (https://doi.org/10.1007/s12079-015-0315-5)

Yu H, Yang C, Jian L, Guo S, Chen R, Li K, Qu F, Tao K, Fu Y, Luo F et al. 2019 Sulfasalazineinduced ferroptosis in breast cancer cells is reduced by the inhibitory effect of estrogen receptor on the transferrin receptor. Oncology Reports 42 826-838. (https://doi.org/10.3892/or.2019.7189)

Zhu Z, Fan X, Lv Y, Lin Y, Wu \& Zeng W 2017 Glutamine protects rabbit spermatozoa against oxidative stress via glutathione synthesis during cryopreservation. Reproduction, Fertility, and Development 29 2183-2194. (https://doi.org/10.1071/RD17020)

Received 2 April 2020

First decision 30 April 2020

Revised Manuscript received 15 July 2020

Accepted 1 September 2020 